\title{
A relaxed multi-direct-forcing immersed boundary-cascaded lattice Boltzmann method accelerated on GPU
}

\author{
Ya Zhang ${ }^{\mathrm{a}, \mathrm{b}}$, Guang Pan ${ }^{\mathrm{a}, \mathrm{b}, *}$, Yonghao Zhang ${ }^{\mathrm{c}}$, Sina Haeri ${ }^{\mathrm{c}, *}$ \\ ${ }^{a}$ School of Marine Science and Technology, Northwestern Polytechnical University, \\ Xi'an, 710072, China \\ ${ }^{b}$ Key Laboratory of Unmanned Underwater Vehicle, Northwestern Polytechnical University, \\ Xi'an, 710072, China \\ ${ }^{c}$ James Weir Fluid Lab, University of Strathclyde, Glasgow, G1 1XJ, UK
}

\begin{abstract}
A new relaxed multi-direct-forcing immersed boundary-cascaded lattice Boltzmann method (MDF IB-CLBM) is proposed in this paper. This new technique improves the efficiency and accuracy of implementing no-slip boundaries on a single graphics processing unit (GPU). The traditional MDF-IBM method essentially solves a linear system iteratively with a relaxation parameter 1.0. By introducing an estimated optimal relaxation parameter, no-slip boundary is fulfilled after only one iteration in a typical case of flow around a fixed cylinder. This is a 7-fold speed-up over the traditional method. Since it is computationally expensive to calculate the estimated optimal relaxation parameter for moving boundaries in every time step, the impact of many factors on the value of the estimated optimal relaxation parameter is investigated. The studied parameters include the discrete delta function, shape of no-slip boundary, the relative distance between several no-slip boundaries, the spacing size between the Lagrangian points, and the relative movement of boundaries over underlying lattices. It is found that the first three factors have a more pronounced influence. Therefore, by considering these three key factors, an appropriate constant
\end{abstract}

\footnotetext{
* Corresponding author

Email addresses: panguang@nwpu.edu.cn (Guang Pan ), sina.haeri@strath.ac.uk (Sina Haeri )
}

Preprint submitted to Computer Physics Communications

October 7, 2019 
relaxation parameter can be determined before starting the simulations. Moreover, the relaxed method provides significant improvement to the traditional MDF-IBM with only a few modifications. The effectiveness of the method is demonstrated by dealing with the flow around a fixed cylinder, the flow past several obstacles, a particle moving in a linear shear flow, and the sedimentation of multiple particles in an enclosure.

Keywords: immersed boundary method, Graphics Processing Unit, fluid-solid interaction, cascaded lattice Boltzmann method

2010 MSC: 00-01, 99-00

\section{Introduction}

The immersed boundary method (IBM) is a powerful tool for fluid-solid interaction problems since first proposed by Peskin [1] in the 1970s for the simulation of blood flow through heart valves. The method enforces the no-slip 5 boundary condition on any complex immersed surface by adding a force density term to the underlying flow equations (such as the Navier-Stokes equations or the lattice Boltzmann equation) which are discretised on a fixed Cartesian grid. IBM is also a popular technique for the fully resolved simulation of a large number of particles moving in fluid since it provides several advantages compared to the body conformal mesh methods [2. For example, by using IBM in fully resolved particle laden flows, one avoids re-meshing of complex physical domains, which is required if a conventional body conformal grid is adopted [25. In IBM, it is vital to find an accurate forcing scheme that can fulfil no-slip boundary condition on surfaces of immersed objects accurately and efficiently.

15 In this work, the multi-direct-forcing immersed boundary method (MDF-IBM) first proposed by Wang et al. [6] is adopted. The body force for implementing no-slip boundary condition is solved iteratively until a given criterion is satisfied.

Other methods proposed for the fully resolved simulation of particle-laden flows include the partially saturated computational cells Noble and Torczynski 20 [7] and the homogenised lattice Boltzmann method Krause et al. [8] which only 
use a fixed Cartesian grid. However, the IBM employs a moving Lagrangian grid to represent the particle, which makes it easier to track the evolution of the immersed surface independently. Despite the availability and development of these methods [7-9], the IBM remains a promising technique for the fully 25 resolved simulation of particle-laden flows.

The lattice Boltzmann method (LBM) is inherently parallelizable [10 12$]$ and can yield the Navier-Stokes equations with second order accuracy [13]. The cascaded lattice Boltzmann method (CLBM), proposed by Geier et al. [14, considerably improves the numerical stability of the LB schemes while preserving the accuracy and simplicity of LBM [15, 16. The MDF-IBM was first coupled with LBM by Suzuki and Inamuro [17. They have validated the MDF-IBM with experimental and theoretical results [17, 18. Minami et al. [19] applied it in the simulation of butterfly and dragonfly-like flapping wings. Then, De Rosis and Lévêque 20] proposed to couple the MDF-IBM with CLBM to deal with fluid-solid interaction problems. The flexibility, stability, and accuracy of the coupled MDF-IB-CLBM scheme have been demonstrated by various fluid-solid interaction problems 20 .

The MDF-IBM has been applied to numerous fluid-solid interaction problems, and hence, there is an ever-increasing interest for improving its accuracy 40 and efficiency. It is especially true while considering the rapid development on heterogeneous computer platforms. A Graphics Processing Unit (GPU) has a massively multi-threaded architecture and is attractive for the parallel computation of data-dense simulations such as fluid-solid interactions. Furthermore, the compute unified device architecture (CUDA) library provided by NVIDIA 45 significantly reduces the development time of a GPU enabled software suite. A very efficient parallel computational performance of LBM as the flow solver could be achieved on GPU [21, 22]. The GPU implementation of MDF-IBM has been analysed in detail by Zhang et al. [23], and in their simulations a maximum speed-up 23.7 of the MDF-IBM on a GPU over that on a single core ${ }_{50}$ of CPU is achieved. However, despite these efforts to accelerate MDF-IBM on GPU [23, 24], the performance bottleneck on GPU remains due to its itera- 
tive nature. To improve the convergence rate and the parallel efficiency of the MDF-IBM, an analysis of the characteristic of MDF-IBM is required.

Different distribution functions have been proposed to improve the accuracy and stability of the MDF-IBM [25, 26]. Moreover, how to choose a suitable Lagrangian mesh size and place a fixed object on lattices are still the subjects of intense research to obtain a better accuracy [18]. The appropriate number of iterations for MDF-IBM has been discussed by Wang et al. [6, Suzuki and Inamuro [17, and Falagkaris et al. 27] to satisfy the required accuracy. Hu et al. [28] introduced an iterative technique to the momentum exchanged-based IBLBM and proved that the distribution of Lagrangian points has little influence on the numerical results. However, there are still some questions regarding the iterative nature of the MDF-IBM that need detailed answers. For example, how to optimise the iteration process to enforce the no-slip boundary condition efficiently, and how the accuracy and efficiency of the method is influenced by different factors such as the distribution functions, Lagrangian mesh size.

The first purpose of this work is to introduce a relaxation technique by analysing the traditional MDF-IBM iteration for the efficient and accurate implementation of no-slip boundaries, and to derive an estimated optimal parameter and validate its convergence rate. The influence of five factors on the estimated optimal relaxation parameter is then studied in the four typical cases. To be specific, we investigated the impact of spacing size between the Lagrangian points considering the flow around a fixed cylinder, the influences of the shape of no-slip boundaries by considering the flow over multiple obstacles, the relative movement over the underlying lattices by considering a particle in a linear shear flow, the relative distance between several no-slip boundaries by considering the sedimentation of particles in an enclosure, and the impact of choice of delta function for all the aforementioned benchmark problems. The calculation for the estimated optimal relaxation parameter should be performed in every time step for simulations involving moving immersed bodies which is computationally expensive and could incur another bottleneck for the parallel computation on GPU. Therefore, determining the impact of various factors facilitates choosing 
an appropriate constant parameter ensuring a balance between the accuracy and efficiency.

The rest of the paper is organised as follows. The IB-CLBM approach and the algorithm of the relaxed MDF-IBM are presented in Section 2. The relaxed MDF-IBM scheme is investigated and validated in Section 3 by considering the flow around a fixed cylinder, the flow over multiple obstacles, the particle in a linear shear flow, and the sedimentation of particles in an enclosure. The effect of different factors is studied in detail. The GPU performance of the relaxed MDF-IBM is also demonstrated in the last case. The concluding remarks are presented in Section 4 .

\section{Numerical method}

\subsection{The immersed boundary-cascaded lattice Boltzmann method}

In CLMB, the computational domain is discretised with a fixed Eulerian grid. The fluid is represented by imaginary particles, which stream along a uniform lattice grid on a fixed reference frame and collide with each other on a moving reference frame. The particles are modelled with a set of distribution functions which evolve according to the following equation:

$$
\mathbf{f}(\mathbf{x}+\mathbf{e} \delta t, t+\delta t)=\mathbf{f}(\mathbf{x}, t)+\mathbf{\Omega}(\mathbf{x}, t)+\mathbf{S} \delta t
$$

where $\mathbf{f}$ is the vector of the particle distribution functions representing the probability of finding a particle at a position $\mathbf{x}$ with a discrete velocity $\mathbf{e}$ at the time $t$. The vector of the discrete collision operators, the vector of the discrete force terms, and the time step are represented by $\boldsymbol{\Omega}, \mathbf{S}$, and $\delta t$, respectively. The discrete velocity set $\mathbf{e}$ of the nine-velocity square lattice model for twodimensional flows (D2Q9) purposed by Qian et al. 29] is

$$
\mathbf{e}=\left[\begin{array}{rrrrrrrrr}
0 & 1 & 0 & -1 & 0 & 1 & -1 & -1 & 1 \\
0 & 0 & 1 & 0 & -1 & 1 & 1 & -1 & -1
\end{array}\right]
$$

The forcing term is incorporated in the central-moment collision operators $\boldsymbol{\Omega}$, which is calculated by $\boldsymbol{\Omega}=\mathbf{K} \cdot \mathbf{k}$, where, $\mathbf{K}$ is a transformation matrix and 
$\mathbf{k}$ is the moment space of the distribution functions $\mathbf{f}[16]$. The discrete forcing term $\mathbf{S}$ is determined by the body force $\mathbf{F}$ [16]. Similar to Guo et al. [30, Premnath and Banerjee [16 implemented the forcing terms in CLBM with a split forcing approach. In this approach, the body forcing term is calculated in two steps, collision and velocity modification. In the evolution from $t$ to $t+\delta t$, the macroscopic density $\rho$ and unmodified velocities $\mathbf{u}^{*}$ can be obtained as follows,

$$
\begin{gathered}
\rho(\mathbf{x}, t)=\sum_{\alpha=0}^{8} f_{\alpha}(\mathbf{x}, t), \\
\rho(\mathbf{x}, t) \mathbf{u}^{*}(\mathbf{x}, t)=\sum_{\alpha=0}^{8} \mathbf{e}_{\alpha} f_{\alpha}(\mathbf{x}, t)+\frac{1}{2} \mathbf{F}(\mathbf{x}, t) \delta t .
\end{gathered}
$$

Then, the colliding and the streaming procedures of CLBM are performed to update the flow field,

$$
\begin{aligned}
& \text { CLBM colliding: } \mathbf{f}^{\prime}(\mathbf{x}, t)=\mathbf{f}(\mathbf{x}, t)+\mathbf{\Omega}(\mathbf{x}, t)+\mathbf{S} \delta t, \\
& \text { CLBM steaming: } \mathbf{f}(\mathbf{x}+\mathbf{e} \delta t, t+\delta t)=\mathbf{f}^{\prime}(\mathbf{x}, t)
\end{aligned}
$$

where $\mathbf{f}^{\prime}$ is the post-collision distribution function.

The kinematic shear viscosity $\nu$ is related to the relaxation parameters $\omega_{\alpha}$ as below,

$$
\begin{gathered}
\nu=c_{s}^{2}\left(\omega_{4,5}^{-1}-0.5\right), \\
\omega_{4}=\omega_{5},
\end{gathered}
$$

where the other relaxation parameters are set to unity [16]. In Eq. (7), $c_{s}=$ $1 / \sqrt{3}$ is the sound speed of the D2Q9 model [13]. The Mach number $M a=u / c_{s}$ should be smaller than 0.1 to limit the compressibility errors in the LBM.

In the IBM, the surface of solid particles is represented by a moving Lagrangian grid. The no-slip boundary is imposed accurately with an appropriate body forcing term $\mathbf{F}_{i b}$ to ensure the fluid velocities $\mathbf{u}$ at the location of Lagrangian points $\mathbf{x}_{l}$ are equal to the boundary velocities $\mathbf{u}_{l}$,

$$
\mathbf{u}\left(\mathbf{x}_{l}, t\right)=\mathbf{u}_{l}\left(\mathbf{x}_{l}, t\right)
$$


In this work, the variables in the Lagrangian frame are represented with a subscript $l$ and the variables in the Eulerian frame do not have any subscript. The two grids communicate via the interpolation and distribution schemes. The fluid velocity at the Lagrangian points $\mathbf{x}_{l}$ is interpolated from the surrounding lattices using

$$
\mathbf{u}\left(\mathbf{x}_{l}, t\right)=\sum_{\mathbf{x}} \mathbf{u}(\mathbf{x}, t) \Phi\left(\mathbf{x}-\mathbf{x}_{l}\right) \delta x^{2},
$$

where $\delta x$ is the lattice spacing. The body force $\mathbf{F}_{i b}$ is obtained by distributing the forces on the Lagrangian points $\mathbf{F}_{i b, l}$ to the background lattices near the solid particle boundaries

$$
\mathbf{F}_{i b}(\mathbf{x}, t)=\sum_{\mathbf{x}_{l}} \mathbf{F}_{i b, l}\left(\mathbf{x}_{l}, t\right) \Phi\left(\mathbf{x}-\mathbf{x}_{l}\right) \delta s,
$$

where $\delta s$ is the spacing between neighbouring Lagrangian points used to represent the solid surface. In Eq. (10), the information of the adjacent lattices around a Lagrangian point are used to calculate the velocity $\mathbf{u}\left(\mathbf{x}_{l}\right)$. The distribution function in Eq. (11) distributes $\mathbf{F}_{i b, l}\left(\mathbf{x}_{l}\right)$ from a Lagrangian point to the surrounding fluid lattices.

The discrete delta function $\Phi$ is

$$
\Phi(x, y)=\frac{1}{\delta x^{2}} \phi\left(\frac{x}{\delta x}\right) \phi\left(\frac{y}{\delta x}\right) .
$$

The interpolation and distribution operations are vital to the global accuracy of the simulation, even though they are only applied in regions near boundaries. Since the choice of delta function can significantly affect the accuracy of simulation [2, some frequently-used delta functions are examined in this work. The following functions are considered,

- a 4-point regularized piecewise function $\phi_{4 r}$,

$$
\phi_{4 r}(r)= \begin{cases}\frac{1}{8}\left(3-2|r|+\sqrt{1+4|r|-4 r^{2}}\right), & |r| \leq 1 \\ \frac{1}{8}\left(5-2|r|-\sqrt{-7+12|r|-4 r^{2}}\right), & 1<|r| \leq 2 \\ 0, & \text { otherwise }\end{cases}
$$


- a 4-point cosine function $\phi_{4 c}$,

$$
\phi_{4 c}(r)= \begin{cases}\frac{1}{4}(1+\cos (\pi|r| / 2)), & |r| \leq 2 \\ 0, & \text { otherwise }\end{cases}
$$

- a smoothed 4-point piecewise function $\phi_{4 s}$ [25],

$$
\phi_{4 s}(r)= \begin{cases}\frac{3}{8}+\frac{\pi}{32}-\frac{r^{2}}{4}, & |r| \leq 0.5 \\ \frac{1}{4}+\frac{1.0-|r|}{8} \sqrt{-2+8|r|-4 r^{2}}-\frac{1}{8} \arcsin (\sqrt{2}(|r|-1)), & 0.5<|r| \leq 1.5 \\ \left.\frac{17}{16}-\frac{\pi}{64}-\frac{3|r|}{4}+\frac{r^{2}}{8}+\frac{|r|-2}{16} \sqrt{(}-14+16|r|-4 r^{2}\right)+\frac{1}{16} \arcsin (\sqrt{2}(|r|-2)), & 1.5<|r| \leq 2.5 \\ 0, & \text { otherwise. }\end{cases}
$$

By adding $\mathbf{F}_{i b}(\mathbf{x})$ to the right-hand side (RHS) of Eq. (4), a corresponding velocity correction $\delta \mathbf{u}(\mathbf{x})$ is obtained as follows:

$$
\rho(\mathbf{x}, t)\left(\mathbf{u}^{*}(\mathbf{x}, t)+\delta \mathbf{u}(\mathbf{x}, t)\right)=\sum_{\alpha=0}^{8} \mathbf{e}_{\alpha} f_{\alpha}(\mathbf{x}, t)+\frac{1}{2}\left(\mathbf{F}(\mathbf{x}, t)+\mathbf{F}_{i b}(\mathbf{x}, t)\right) \delta t .
$$

Thus, $\mathbf{F}_{i b}(\mathbf{x})$ can be calculated from $\delta \mathbf{u}(\mathbf{x})$ by subtracting Eq. (4) from Eq. (16) as

$$
\mathbf{F}_{i b}(\mathbf{x}, t)=\frac{2 \rho \delta \mathbf{u}(\mathbf{x}, t)}{\delta t},
$$

and $\mathbf{F}_{i b, l}\left(\mathbf{x}_{l}\right)$ can be expressed in a similar form of

$$
\mathbf{F}_{i b, l}\left(\mathbf{x}_{l}, t\right)=\frac{2 \rho \delta \mathbf{u}_{l}\left(\mathbf{x}_{l}, t\right)}{\delta t} .
$$

Then, the distribution operation in Eq. 18 is transformed into

$$
\delta \mathbf{u}(\mathbf{x}, t)=\sum_{\mathbf{x}_{l}} \delta \mathbf{u}_{l}\left(\mathbf{x}_{l}, t\right) \Phi\left(\mathbf{x}-\mathbf{x}_{l}\right) \delta s,
$$

and the no-slip boundary is satisfied when

$$
\mathbf{u}_{l}\left(\mathbf{x}_{l}, t\right)=\sum_{\mathbf{x}}\left(\mathbf{u}^{*}(\mathbf{x}, t)+\delta \mathbf{u}(\mathbf{x}, t)\right) \Phi\left(\mathbf{x}-\mathbf{x}_{l}\right) \delta x^{2} .
$$

The total hydrodynamic force $\mathbf{F}_{h}$ and torque $\mathbf{T}_{h}$ exerted on an object are then calculated by

$$
\mathbf{F}_{h}(\mathbf{x}, t)=-\sum_{\mathbf{x}_{l}} \mathbf{F}_{i b, l}\left(\mathbf{x}_{l}, t\right) \delta s+\mathbf{F}_{i n}
$$




$$
\mathbf{T}_{h}(\mathbf{x}, t)=-\sum_{\mathbf{x}_{l}}\left(\mathbf{x}_{l}-\mathbf{X}\right) \times \mathbf{F}_{i b, l}\left(\mathbf{x}_{l}, t\right) \delta s+\mathbf{T}_{i n},
$$

where $\mathbf{X}$ is the mass centre of the object, $\mathbf{F}_{\text {in }}$ and $\mathbf{T}_{i n}$ represent the effect of internal mass [31.

\subsection{The traditional MDF-IBM}

Two algorithms used by De Rosis and Lévêque 20] and Zhang et al. 23] are available for the MDF-IB-CLBM. The first algorithm used by De Rosis and Lévêque [20] is directly based on Eqs. (19) and 20],

$$
\begin{aligned}
\text { Interpolation: } \mathbf{u}^{k}\left(\mathbf{x}_{l}, t\right) & =\sum_{\mathbf{x}} \mathbf{u}^{k}(\mathbf{x}, t) \Phi\left(\mathbf{x}-\mathbf{x}_{l}, t\right) \delta x^{2} \\
\delta \mathbf{u}_{l}^{k}\left(\mathbf{x}_{l}, t\right) & =\delta \mathbf{u}_{l}^{k-1}\left(\mathbf{x}_{l}, t\right)+\mathbf{u}_{l}\left(\mathbf{x}_{l}, t\right)-\mathbf{u}^{k}\left(\mathbf{x}_{l}, t\right) \\
\text { Distribution: } \delta \mathbf{u}^{k}(\mathbf{x}, t) & =\sum_{\mathbf{x}_{l}} \delta \mathbf{u}_{l}^{k}\left(\mathbf{x}_{l}, t\right) \Phi\left(\mathbf{x}-\mathbf{x}_{l}\right) \delta s \\
\mathbf{u}^{k+1}(\mathbf{x}, t) & =\mathbf{u}^{*}(\mathbf{x}, t)+\delta \mathbf{u}^{k}(\mathbf{x}, t)
\end{aligned}
$$

where $k$ is the loop counter. The boundary forces $\mathbf{F}_{i b, l}\left(\mathbf{x}_{l}\right)$ and $\mathbf{F}_{i b}(\mathbf{x})$ can be computed from Eq. (17) and Eq. (18), respectively. In Eq. 26), both the values of $\mathbf{u}^{*}(\mathbf{x})$ and $\mathbf{u}^{k+1}(\mathbf{x})$ should be stored. Considering the limited global memory on GPU, Zhang et al. 23] used a cumulative correction procedure to reduce memory consumption,

$$
\delta \mathbf{u}_{l}^{k}\left(\mathbf{x}_{l}, t\right)=\sum_{0}^{k} \Delta \mathbf{u}_{l}^{k}\left(\mathbf{x}_{l}, t\right)
$$


The iteration is conducted following Eqs. 28 - 33 as

$$
\begin{gathered}
\text { Interpolation: } \mathbf{u}^{k}\left(\mathbf{x}_{l}, t\right)=\sum_{\mathbf{x}} \mathbf{u}^{k}(\mathbf{x}, t) \Phi\left(\mathbf{x}-\mathbf{x}_{l}, t\right) \delta x^{2}, \\
\Delta \mathbf{u}_{l}^{k}\left(\mathbf{x}_{l}, t\right)=\mathbf{u}_{l}\left(\mathbf{x}_{l}, t\right)-\mathbf{u}^{k}\left(\mathbf{x}_{l}, t\right), \\
\mathbf{F}_{i b, l}^{k}\left(\mathbf{x}_{l}, t\right)=\mathbf{F}_{i b, l}^{k-1}\left(\mathbf{x}_{l}, t\right)+\frac{2 \rho \Delta \mathbf{u}_{l}\left(\mathbf{x}_{l}, t\right)}{\delta t} ; \\
\text { Distribution: } \Delta \mathbf{u}^{k}(\mathbf{x}, t)=\sum_{\mathbf{x}_{l}} \Delta \mathbf{u}_{l}^{k}\left(\mathbf{x}_{l}, t\right) \Phi\left(\mathbf{x}-\mathbf{x}_{l}\right) \delta s \\
\mathbf{u}^{k+1}(\mathbf{x}, t)=\mathbf{u}^{k}(\mathbf{x}, t)+\Delta \mathbf{u}^{k}(\mathbf{x}, t), \\
\mathbf{F}_{i b}^{k}(\mathbf{x}, t)=\mathbf{F}_{i b}^{k-1}(\mathbf{x}, t)+\frac{2 \rho \Delta \mathbf{u}(\mathbf{x}, t)}{\delta t} .
\end{gathered}
$$

Both algorithms require a relatively large number of iterations, otherwise they cannot fulfil the no-slip boundary with high precision. However, the multiple invocations of interpolation operation using Eqs. (23) and $(28)$, and the distribution operation using Eqs. 25) and (31) cause a significant overhead on the computations and reduce the parallel efficiency on the GPU.

When substituting Eqs. 23), 26 and 25 into Eq. 24, and Eqs. (28), (32) and (31) into Eq. (29), the two MDF-IBM algorithms, Eqs. (23)-26) and Eqs. 28-33), can be expressed respectively as $\delta \mathbf{u}_{l}^{k}\left(\mathbf{x}_{l}\right)=\delta \mathbf{u}_{l}^{k-1}\left(\mathbf{x}_{l}\right)+\left\{\mathbf{u}_{l}\left(\mathbf{x}_{l}\right)-\sum_{\mathbf{x}}\left[\mathbf{u}^{*}(\mathbf{x})+\sum_{\mathbf{x}_{m}} \delta \mathbf{u}_{m}^{k-1}\left(\mathbf{x}_{m}\right) \Phi\left(\mathbf{x}-\mathbf{x}_{m}\right) \delta s_{m}\right] \Phi\left(\mathbf{x}-\mathbf{x}_{l}\right) \delta x^{2}\right\}$,

$\Delta \mathbf{u}_{l}^{k}\left(\mathbf{x}_{l}\right)=\mathbf{u}_{l}\left(\mathbf{x}_{l}\right)-\sum_{\mathbf{x}}\left[\mathbf{u}^{k-1}(\mathbf{x})+\sum_{\mathbf{x}_{m}} \Delta \mathbf{u}_{m}^{k-1}\left(\mathbf{x}_{m}\right) \Phi\left(\mathbf{x}-\mathbf{x}_{m}\right) \delta s_{m}\right] \Phi\left(\mathbf{x}-\mathbf{x}_{l}\right) \delta x^{2}$.

In Eq. 27), $\Delta \mathbf{u}_{l}^{k}\left(\mathbf{x}_{l}\right)$ is given by

$$
\Delta \mathbf{u}_{l}^{k}\left(\mathbf{x}_{l}\right)=\delta \mathbf{u}_{l}^{k}\left(\mathbf{x}_{l}\right)-\delta \mathbf{u}_{l}^{k-1}\left(\mathbf{x}_{l}\right)
$$

Moreover, the fluid velocity $\mathbf{u}^{k-1}(\mathbf{x})$ in Eq. 35 is obtained from the multidistribution of $\Delta \mathbf{u}_{m}\left(\mathbf{x}_{m}\right)$,

$\mathbf{u}^{k-1}(\mathbf{x})=\mathbf{u}^{*}(\mathbf{x})+\sum_{\mathbf{x}_{m}} \Delta \mathbf{u}_{m}^{0}\left(\mathbf{x}_{m}\right) \Phi\left(\mathbf{x}-\mathbf{x}_{m}\right) \delta s_{m}+\ldots+\sum_{\mathbf{x}_{m}} \Delta \mathbf{u}_{m}^{k-2}\left(\mathbf{x}_{m}\right) \Phi\left(\mathbf{x}-\mathbf{x}_{m}\right) \delta s_{m}$. 
Substituting Eqs. (36) and (37) in Eq. (35), it turns out that Eqs. (34) and (35) are in fact equivalent and follow the same linear structure

$$
\mathbf{X}^{k}=\mathbf{X}^{k-1}+\left(\mathbf{b}-\mathbf{A} \mathbf{X}^{k-1}\right),
$$

where $\mathbf{A}$ is a sparse metrics of size $N \times N, \mathbf{X}$ and $\mathbf{b}$ are vectors of size $N$, and $N$ is the number of Lagrangian points. In Eq. (38), the components of matrix $\mathbf{A}$ and vectors $\mathbf{X}$ and $\mathbf{b}$ are given as follows,

$$
\begin{aligned}
& \mathbf{A}_{l m}=\sum_{\mathbf{x}} \Phi\left(\mathbf{x}-\mathbf{x}_{m}\right) \Phi\left(\mathbf{x}-\mathbf{x}_{l}\right) \delta s_{m} \delta x^{2}, \\
& \mathbf{X}_{m}=\delta \mathbf{u}_{m}\left(\mathbf{x}_{m}\right) \\
& \mathbf{b}_{l}=\mathbf{u}_{l}\left(\mathbf{x}_{l}\right)-\sum_{\mathbf{x}} \mathbf{u}^{*}(\mathbf{x}) \Phi\left(\mathbf{x}-\mathbf{x}_{l}\right) \delta x^{2} .
\end{aligned}
$$

This analysis shows that the MDF-IBM is an explicit iteration scheme to the linear system: $\mathbf{A X}=\mathbf{b}$. Wu and $\mathrm{Shu} 32$ solves the equation with a direct method requiring matrix inversions, which makes it infeasible for simulations involving multiple moving particles due to the computational complexity of matrix inversions. Here the MDF-IBM explicit iteration for solving Eq. 38 will be improved to obtain a faster convergence rate.

\subsection{The relaxed $M D F-I B M$}

In the traditional MDF-IBM, Eq. (38) takes the iteration form as

$$
\mathbf{X}^{k}=\mathbf{B X}^{k-1}+\mathbf{b}
$$

where $\mathbf{B}=\mathbf{I}-\mathbf{A}$ is the iteration matrix, $\mathbf{I}$ is the $N \times N$ identity matrix. Following Hu et al. [28], the Richardson iteration with the relaxation parameter $\omega$ is introduced to accelerate the convergence rate,

$$
\mathbf{X}^{k}=(\mathbf{I}-\omega \mathbf{A}) \mathbf{X}^{k-1}+\omega \mathbf{b}=\mathbf{X}^{k-1}+\omega\left(\mathbf{b}-\mathbf{A} \mathbf{X}^{k-1}\right),
$$

where $\mathbf{B}=\mathbf{I}-\omega \mathbf{A}$. It is noteworthy that the traditional MDF-IBM uses the Richardson iteration with a relaxation parameter $\omega=1.0 . \omega$ is introduced into Eqs. 24) and (29),

$$
\delta \mathbf{u}_{l}^{k}\left(\mathbf{x}_{l}\right)=\delta \mathbf{u}_{l}^{k-1}\left(\mathbf{x}_{l}\right)+\omega\left(\mathbf{u}_{l}\left(\mathbf{x}_{l}\right)-\mathbf{u}^{k-1}\left(\mathbf{x}_{l}\right)\right),
$$




$$
\Delta \mathbf{u}_{l}^{k}\left(\mathbf{x}_{l}\right)=\omega\left(\mathbf{u}_{l}\left(\mathbf{x}_{l}\right)-\mathbf{u}^{k-1}\left(\mathbf{x}_{l}\right)\right)
$$

while the other steps remain the same. Therefore, for both MDF schemes only limited modification is required to transform the traditional MDF-IBM to a relaxed scheme. The convergence rate can be significantly increased with a relaxation parameter $\omega$, and more accurate results can be obtained after a limited number of iterations.

As $\mathbf{A}$ is a symmetric positive-definite matrix, the optimal relaxation parameter is $\omega_{\mathrm{opt}}=2 /\left(\lambda_{\max }+\lambda_{\min }\right)$ and the convergent range is $0<\omega<2 \lambda_{\max }^{-1}$, where $\lambda$ is an eigenvalue of $\mathbf{A}$. However, it is computationally expensive to calculate the eigenvalues of $\mathbf{A}$ especially when the number of Lagrangian points $N$ is large. There is the relationship between the maximum eigenvalue $\lambda_{\max }$ and the infinity norm $\|\mathbf{A}\|_{\infty}, \lambda_{\max } \leq\|\mathbf{A}\|_{\infty}$. Then an estimated optimal value is obtained,

$$
\tilde{\omega}=\|\mathbf{A}\|_{\infty}^{-1} \leq \lambda_{\max }^{-1} \leq \omega_{\mathrm{opt}},
$$

and a convergent range

$$
0<\omega<2\|\mathbf{A}\|_{\infty}^{-1}
$$

The closer the value of $\omega$ is to the estimated optimal relaxation parameter ${ }_{130}\|\mathbf{A}\|_{\infty}^{-1}$, the faster the convergence rate is.

To examine the imposition of the no-slip boundary, the fluid velocity at the Lagrangian points is interpolated with Eq. 28) after the relaxed iteration. The numerical error $E$, to assess the quality of the no-slip boundary quantitatively, is defined as

$$
E=\frac{\sum_{N}\left\|\mathbf{u}_{l}\left(x_{l}\right)-\mathbf{u}^{N_{i t r}}\left(x_{l}\right)\right\|}{N}
$$

where $N_{i t r}$ is the number of iterations. The algorithm of the relaxed MDF IB-CLBM is listed as follows.

1. The distribution functions $\mathbf{f}$ at each fluid lattice are initialized as the equilibrium distribution state.

2. Distribute Lagrangian points $\mathbf{x}_{l}$ evenly on the predefined boundaries and determine $\delta s$. 
3. Calculate $\|\mathbf{A}\|_{\infty}^{-1}$ and determine the relaxation parameter $\omega$.

4. CLBM: Calculate the macroscopic fluid variables with Eq. (3) and Eq. (4).

5. The relaxed MDF-IBM:

140

(a) Update the relaxation parameter $\omega, \omega=\|\mathbf{A}\|_{\infty}^{-1}$.

(b) Set $k=0, \mathbf{u}^{0}(\mathbf{x})=\mathbf{u}^{*}(\mathbf{x}), \mathbf{F}_{i b, l}\left(\mathbf{x}_{l}\right)=0$, and $\mathbf{F}_{i b}(\mathbf{x})=0$.

(c) Interpolation in Eqs. (28), (45) and (30).

(d) Distribution in Eqs. (31)- 33).

(e) Advance $k$ to $k+1$ and repeat step $5 \mathrm{c}$ to $5 \mathrm{~d}$ while $k<N_{i t r}$.

6. Calculate the boundary numerical error $E$ with Eq. 487.

7. Obtain the hydrodynamic force $\mathbf{F}_{h}$ and torque $\mathbf{T}_{h}$ according to Eqs. 21) and 22

8. Update the locations and velocities of Lagrangian points of moving boundaries.

9. Perform CLBM colliding and streaming according to Eq. (5) and Eq. (6).

10. Advance to time step $n+1$ and repeat steps 4 to 9 until the simulation is finished.

When dealing with the stationary boundaries, the matrix $\mathbf{A}$ needs to be calculated once at Step 3 and no further update is required. However, for moving objects the position of Lagrangian points should be updated at Step 8 at every time step. Therefore, theoretically, $\mathbf{A}$ should be solved in every evolution at Step 5a, which will increase the computational effort. Especially when the number of Lagrangian points $N$ is quite large, the limited global memory available on a GPU may not be enough to store the matrix A. For the same reason, in this work the iteration does not follow the form of Eq. 433 that includes the matrix-vector operations.

A value of $\omega$ that is close to the estimated optimal relaxation parameter $\|\mathbf{A}\|^{-1}$ could also reach a fast convergence rate. Thus, an appropriate constant $\omega$ can help to avoid the frequent update of $\mathbf{A}$ at Step $5 \mathrm{a}$ for moving objects. The investigation of the influence of factors including the delta functions, the shape of boundary, the relative location between boundaries, the spacing between 
Lagrangian points, and the relative movement of boundaries over underlying lattice, on the value of $\|\mathbf{A}\|^{-1}$ will help to explore the possibility of employing such a constant relaxation parameter. In the following content, the estimated optimal relaxation parameter is used when $\omega=\|\mathbf{A}\|^{-1}$, the appropriate constant relaxation parameter is used when $\omega$ equals to a certain constant, except $\omega=1.0$, which refers to the traditional MDF-IBM.

\subsection{The GPU implementation details}

The algorithm on GPU is programmed with $\mathrm{C}$ language under the Compute Unified Device Architecture (CUDA). In the CUDA architecture, GPU is regarded as the device and the code running on it is launched by the host (CPU). The GPU implementations of the LBM and the traditional MDF-IBM have been fully discussed in previous literature [21,24, where Steps 4, 5c, 5d, 7 and 9 have been analysed extensively. Moreover, the introduction of the relaxation parameter in Eqs. (44) and (45) has little influence on the GPU implementation and performance of Steps $5 \mathrm{c}$ and $5 \mathrm{~d}$. Therefore, the focus here is to calculate an estimated optimal relaxation parameter $\|\mathbf{A}\|_{\infty}^{-1}$ on a GPU during Steps 3 and 5a. All the involved variables are stored on the device global memory as onedimensional arrays to facilitate the coalesced memory access. The elements of $\mathbf{A}$ are stored linearly in a dense vector format. The frequently accessed constants, such as $\delta x$, are stored in the device constant memory.

In the computation of the matrix $\mathbf{A}$ on a GPU, every thread deals with one element according to Eq. (39). The number of threads launched is determined by the square of the number of Lagrangian points $N^{2}$. Due to the two-level management of threads, the threads are organized into two-dimensional blocks with sizes, BLOCKX and _BLOCKY. The number of threads in a block is BLOCKX*_BLOCKY. The element $\mathbf{A}_{l m}$ is not zero only when the distance between the two Lagrangian points satisfies $\left\|\mathbf{X}_{l}-\mathbf{X}_{m}\right\|<4 \delta x$. The value of non-zero elements is related to the overlap zone of two Lagrangian points as demonstrated in Fig. 1. Thus, the support lattice nodes of the Lagrangian point $\mathbf{x}_{l}$ can be included in the calculation to reduce the calculations done by a single 


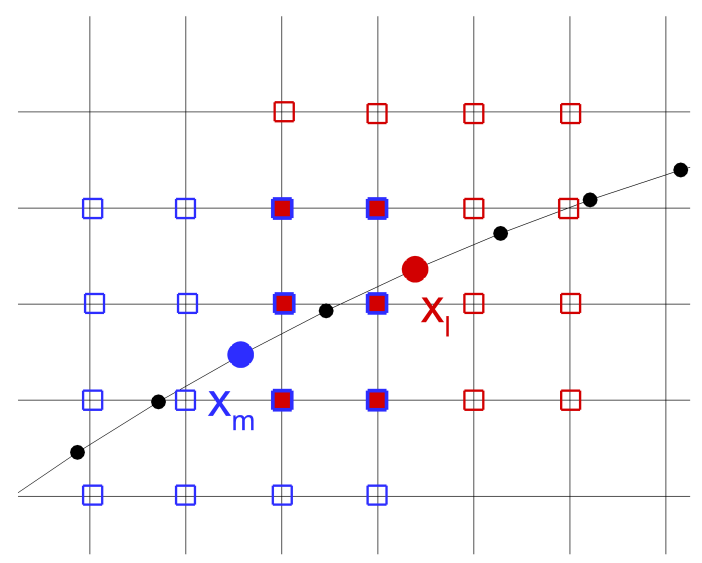

Figure 1: Schematic of the support lattices of Lagrangian points, Lagrangian points: the support lattice nodes: $\square_{\text {and }} \square$; the overlap zone:

thread. The pseudo-code section A1 for calculating the matrix $\mathbf{A}$ is illustrated in Algorithm 1

As the infinite norm $\|\mathbf{A}\|_{\infty}=\max _{l} \sum_{m} A_{l m}$, the row-sum $\sum_{m} A_{l m}$, which is the sum of all elements in the $l$-th row, should be computed first. The kernel RowSum_reduce() performing a parallel sum-reduction operation is used as presented in the pseudo-code section A2 in Algorithm 2. The infinite norm $\|\mathbf{A}\|_{\infty}$ is then obtained with a parallel comparison-reduction operation over the obtained row-sums (the kernel ColMax_reduce() in Algorithm 3). The threads inside a block in the function RowSum_reduce() are organized with a one-dimensional form with sizes, _BLOCK_1D, and the blocks are organised into a two-dimensional grid, while both the blocks and the threads inside in ColMax_reduce() are indexed with a one-dimensional form. The memories mem_device and mem_host are allocated on the device and the host, respectively. Their sizes are $B x \times N \times$ sizeof(double) in Algorithm 2, and Bx $\times$ sizeof(double) in Algorithm 3. The parallel comparison-reduction operation implemented in the kernel ColMax_reduce() is illustrated in Fig. 2, where the sequential addressing is used to achieve a coalesced read/write. In the parallel sum-reduction operation of RowSum_reduce(), the comparison between elements is replaced with 


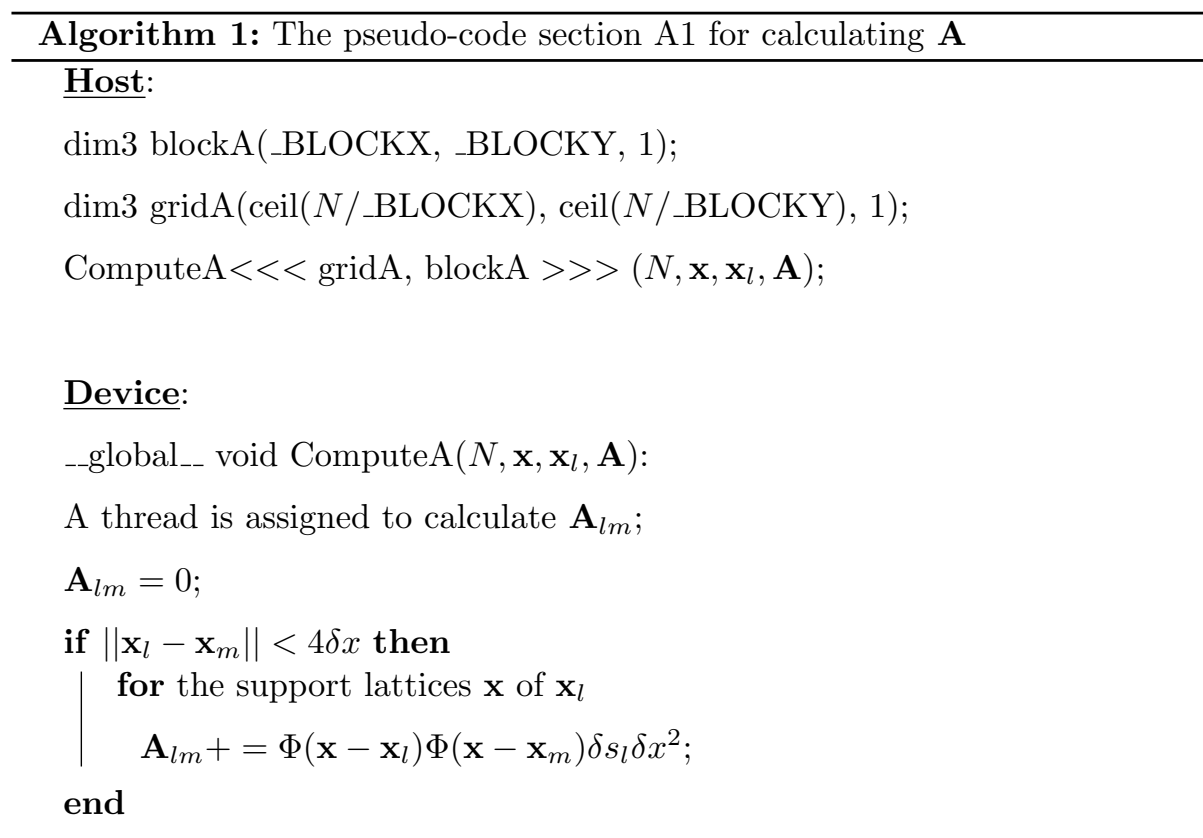

addition.

\section{Numerical simulations}

The relaxed MDF-IBM will be validated considering several numerical examples. In Section 3.1, the flow over a fixed cylinder is simulated and its results are compared with those obtained from the traditional MDF-IBM. The case of flow past several obstacles of different shapes is presented in Section 3.2 . In Section 3.3 a particle in a shear flow is considered to study the variation of $\|\mathbf{A}\|{ }_{\infty}{ }^{-1}$ as the particle moves over the underlying lattices. In Section 3.4, the influence of the relative locations between moving boundaries on $\|\mathbf{A}\|_{\infty}{ }^{-1}$ is revealed in the simulation of the sedimentation of multiple particles. These cases will help ${ }_{225}$ us identify the contribution of different factors to the variation of $\|\mathbf{A}\| \|_{\infty}^{-1}$ and focus on the key to determining an appropriate constant relaxation parameter $\omega$ in order to achieve a high convergence rate by ensuring $\omega$ close to $\|\mathbf{A}\|_{\infty}{ }^{-1}$. In Section 3.4 the parallel computational performance of the relaxed MDF-IBM on a single GPU is presented and compared with the traditional MDF-IBM. 

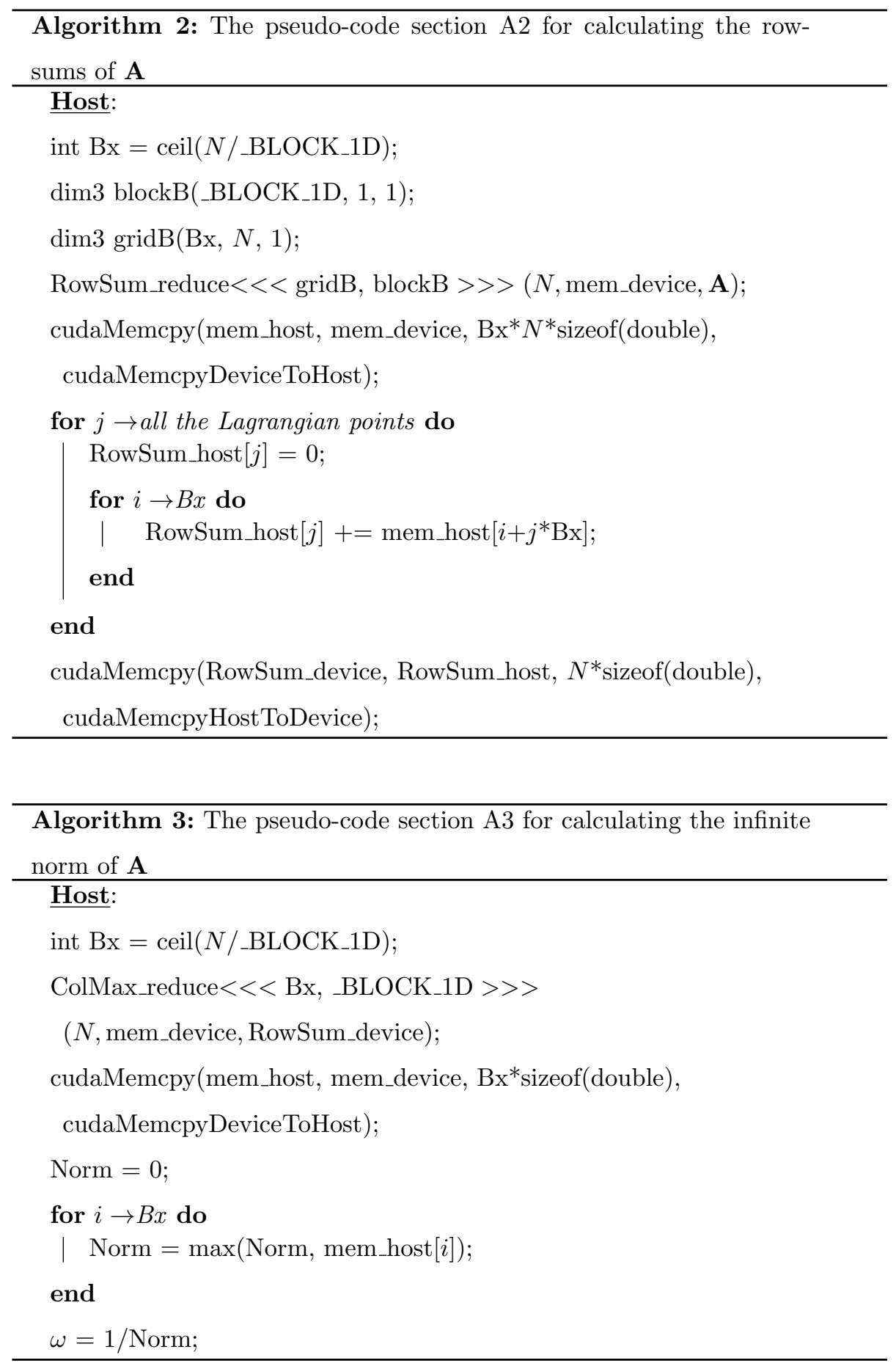


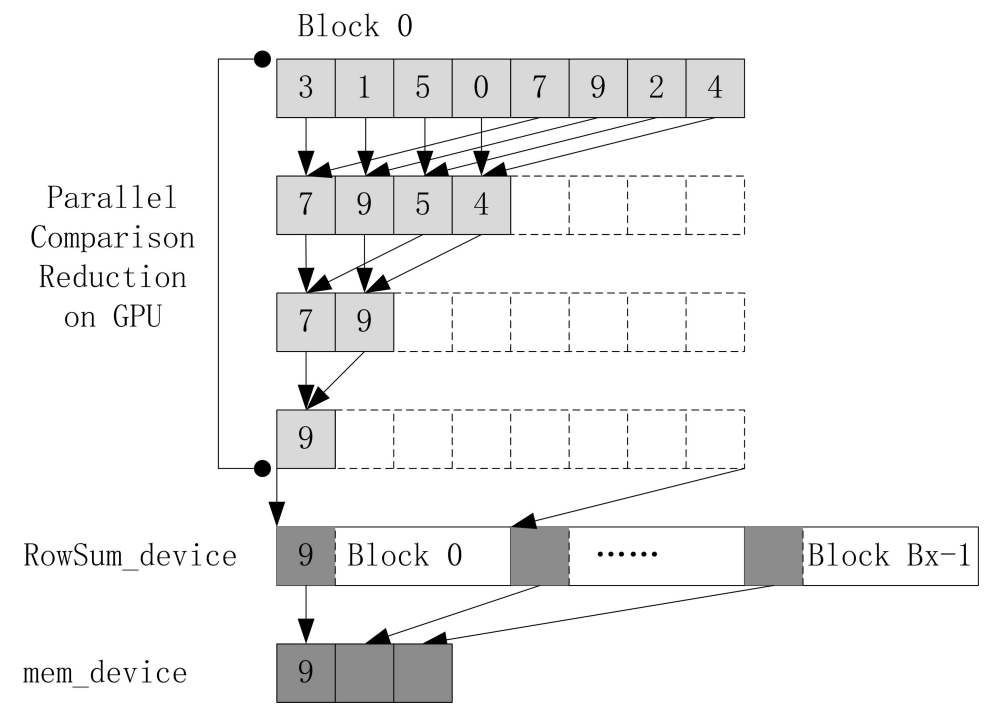

Figure 2: The illustration of parallel comparison-reduction operation implemented in the kernel ColMax_reduce().

\subsection{Flow over a fixed cylinder}

A circular cylinder with diameter $D$ is fixed at the location $(8 D, 10 D)$ of the computational domain of size $28 D \times 20 D$. The fluid density is $\rho=1.0$ in lattice units. A zero-gradient boundary condition is applied at the upper, the lower, and the outlet of the channel while a velocity inflow boundary condition with ${ }_{235} U_{0}=0.05$ in lattice units at the inlet. The Reynolds number is calculated with $R e=U_{0} D / \nu$. There are 50 lattice spacings over the particle's diameter. The drag and the lift coefficients respectively, $C_{d}=2 F_{d} / \rho U_{0}^{2} D$ and $C_{l}=2 F_{l} / \rho U_{0}^{2} D$, are calculated for comparison. The Strouhal number $S t=f_{q} D / U_{0}$ depends on the frequency of the vortex shedding $f_{q}$, which is obtained by applying a fast Fourier transform to $C_{l}$ curves.

In the studies of Suzuki and Inamuro [17] and Suzuki et al. 18] with the traditional MDF-IBM, the number of iterations $N_{i t r}$ is usually taken as 5 , which is assumed sufficient to accurately enforce the no-slip boundary condition. The spacing size $\delta s$ between the Lagrangian points should be chosen to avoid unnecessary computational overhead induced by an excessive meshing and eliminate 
Table 1: Effect of delta functions at $R e=20$.

\begin{tabular}{llllll}
\hline Delta functions & $\phi_{4 r}$ & $\phi_{4 c}$ & $\phi_{4 s}$ & He and Doolen [34] & Calhoun [35] \\
\hline$\omega=\|\mathbf{A}\|_{\infty}{ }^{-1}$ & 2.593 & 2.605 & 2.801 & & \\
$E$ & $3.364 \mathrm{e}-6$ & $1.331 \mathrm{e}-5$ & $6.497 \mathrm{e}-6$ & & \\
$C_{d}$ & 2.163 & 2.163 & 2.170 & 2.152 & 2.19 \\
\hline
\end{tabular}

fluid flux through the no-slip boundaries [33. In this case, the influence of $\delta s$ and delta functions on the value of $\|\mathbf{A}\|_{\infty}{ }^{-1}$, and $\omega$ and $N_{i t r}$ on accuracy of the no-slip boundary will be studied. Here all the Lagrangian points are distributed evenly on the surface of the cylinder with $\delta s \approx(0.5,1.0,1.5) \delta x$.

Table 1 shows the effect of different delta functions on the drag coefficient $C_{d}$, where $N_{i t r}=5$ and $\delta s=1.0 \delta x$ at $R e=20$. The drag coefficients $C_{d}$ are compared with each other and the published results. It shows that $C_{d}$ values of the delta functions $\Phi_{4 r}$ and $\Phi_{4 c}$ are similar and that of $\Phi_{4 s}$ is larger, and all of them are within the range of results from He and Doolen [34] and Calhoun 255 35]. However, the delta function $\Phi_{4 r}$ satisfies the no-slip boundary condition with the highest accuracy. The grid convergence study is conducted as shown in Table 2. Evidently, the numerical error $E$ and the drag efficient $C_{d}$ converge as the grid resolution increases. The experimental order of convergence (EOC) [ $[$, $\operatorname{EOC}\left(D_{1}, D_{2}\right)=\log \left(E_{1} / E_{2}\right) / \log \left(D_{2} / D_{1}\right)$, is computed to show an approximate linear convergence, which is close to the result of Krause et al. [8]. Fig. 3] depicts the variation of $\|\mathbf{A}\|^{-1}$ with $\delta s$. It seems that $\|\mathbf{A}\|_{\infty}{ }^{-1}$ varies in a small interval, i.e. $(2.58,2.61)$ for $\Phi_{4 s}$, and $\Phi_{4 r}$ and $(2.78,2.80)$ for $\Phi_{4 s}$, with the average values 2.6 and 2.8 , respectively.

Simulations are also performed at $R e=200$ to further verify the influence of $\delta s$ and $N_{i t r}$ on $E, C_{d}$, and $C_{l}$ when the average relaxation parameter $\omega=2.6$ is employed with $\Phi_{4 r}$. Table 3 shows the numerical error $E$ in the no-slip boundary conditions of the relaxed and traditional MDF-IBM. By increasing $N_{i t r}$, generally the error $E$ is reduced for both schemes. The parameters $C_{d}, C_{l}$, and $S t$ remain constant as $N_{i t s}$ is increased for the relaxed approach whereas 
Table 2: Grid convergence study with $R e=20, U_{0}=0.05$, and $\phi_{4 r}$.

\begin{tabular}{llllll}
\hline$D / \delta x$ & $\nu$ & $\omega=\|\mathbf{A}\|_{\infty}{ }^{-1}$ & $C_{d}$ & $E$ & $\operatorname{EOC}$ \\
\hline 20 & 0.05 & 2.587 & 2.205 & $8.510 \mathrm{e}-6$ & $\operatorname{EOC}(20,30): 1.4$ \\
30 & 0.075 & 2.589 & 2.181 & $4.782 \mathrm{e}-6$ & $\operatorname{EOC}(30,40): 0.7$ \\
40 & 0.1 & 2.592 & 2.172 & $3.896 \mathrm{e}-6$ & $\operatorname{EOC}(40,50): 0.7$ \\
50 & 0.125 & 2.593 & 2.163 & $3.364 \mathrm{e}-6$ & Average: 1.0 \\
\hline
\end{tabular}

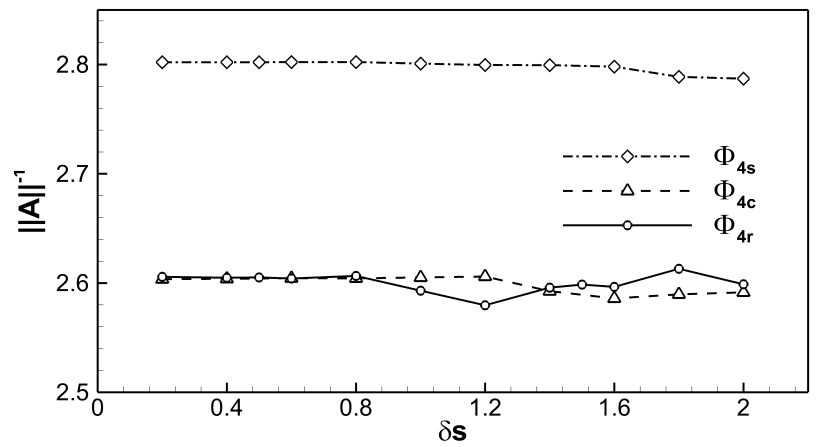

Figure 3: Variation of the value of $\|\mathbf{A}\|^{-1}$ with the spacing $\delta s$. 
Table 3: Effect of $N_{i t r}$ and $\delta s$ for $R e=200$.

\begin{tabular}{llllllllllll}
\hline \multicolumn{1}{c}{ The traditional MDF-IBM $(\omega=1.0)$} & \multicolumn{4}{c}{ The relaxed MDF-IBM $(\omega=2.6)$} \\
\hline$N_{i t r}$ & $\delta s$ & $E$ & $C_{d}$ & $C_{l}$ & $S t$ & $E$ & $C_{d}$ & $C_{l}$ & $S t$ \\
1 & 0.5 & & & & & $1.131 \mathrm{e}-5$ & 1.411 & 0.830 & 0.200 \\
1 & 1.0 & $1.008 \mathrm{e}-4$ & 1.424 & 0.844 & 0.200 & $1.089 \mathrm{e}-5$ & 1.411 & 0.830 & 0.200 \\
1 & 1.5 & & & & & $6.171 \mathrm{e}-6$ & 1.411 & 0.831 & 0.200 \\
2 & 1.0 & $5.245 \mathrm{e}-5$ & 1.418 & 0.840 & 0.200 & $9.317 \mathrm{e}-6$ & 1.411 & 0.830 & 0.200 \\
5 & 1.0 & $1.553 \mathrm{e}-5$ & 1.412 & 0.837 & 0.200 & $7.738 \mathrm{e}-6$ & 1.411 & 0.830 & 0.200 \\
7 & 1.0 & $1.053 \mathrm{e}-5$ & 1.411 & 0.830 & 0.200 & & & & \\
\hline
\end{tabular}

270 a decreasing trend is observed for the traditional MDF-IBM. It seems that an acceptable no-slip boundary can be obtained with a smaller number of iterations $N_{i t r}$ and an arbitrary Lagrangian point spacing $\delta s$ within the range $(0.5-1.5) \delta x$. The error $E$ of the traditional MDF with $N_{i t r}=7$ is comparable with that of the relaxed MDF with $N_{i t r}=1$. Therefore, it is reasonable to ignore the influence of $275 \delta s$ on the estimated optimal relaxation parameter and use an average relaxation value. Moreover, it has been validated that a reduced iteration time $N_{i t r}$ is needed by the relaxed MDF-IBM in the simulations of steady and unsteady flow over a fixed cylinder.

Fig. 4 shows the streamlines when flow reaches the steady state for $R e=20$ and $R e=200$, where the streamline behaves differently depending on the Reynolds number. At the low Reynolds number $R e=20$, a pair of symmetrical vortexes appears behind the cylinder, while at $R e=200$, a von karman vortex street is observed at the vorticity contour displayed in Fig. 5 . Fig. 6 demonstrates the pressure distribution on the surface of the fixed cylinder at $R e=20$, which is in an excellent agreement with that from Wu and Shu [32]. Evolution of the drag and the lift coefficients for $R e=200$ and $R e=1000$ with a dimensionless time $t^{*}=t U_{0} / D$ are displayed in Fig. 7. Tables 4 and 5 compare the characteristic parameters with the previous numerical simulations. 


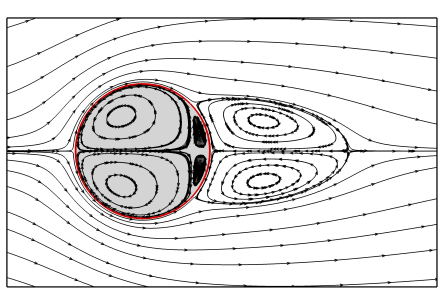

(a) $R e=20$

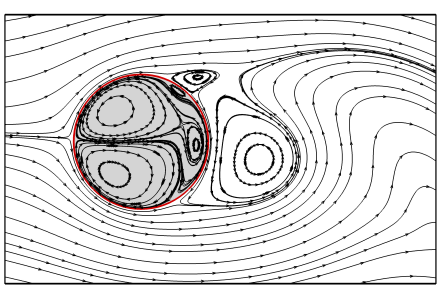

(b) $R e=200$

Figure 4: The streamlines of flow over a fixed cylinder at $R e=20$ and $R e=200$.

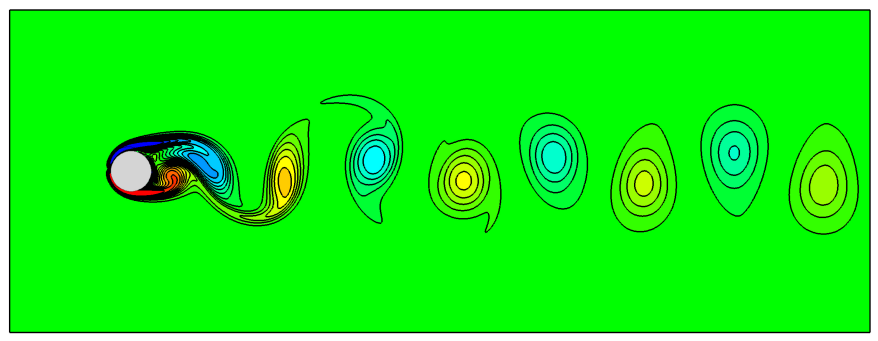

Figure 5: The vorticity contour of flow over a fixed cylinder at $R e=200$.

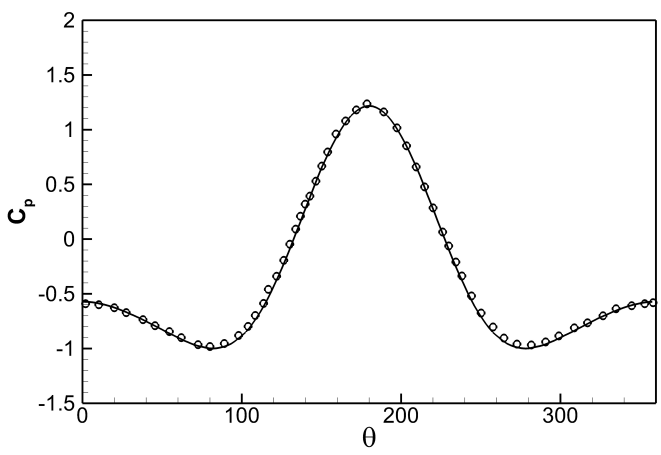

Figure 6: The pressure distribution on the surface of the fixed cylinder at $R e=20$, results from $\mathrm{Wu}$ and Shu [32]: O; present results:

Table 4: Comparison of coefficients $C_{d}$ and $S t$ for $R e=200$.

\begin{tabular}{lllll}
\hline & The relaxed MDF-IBM & Braza et al. [36] & Benson et al. [37] & Le et al. [38] \\
\hline$C_{d}$ & 1.41 & 1.38 & 1.45 & 1.38 \\
$S t$ & 0.20 & 0.19 & 0.193 & 0.192 \\
\hline
\end{tabular}




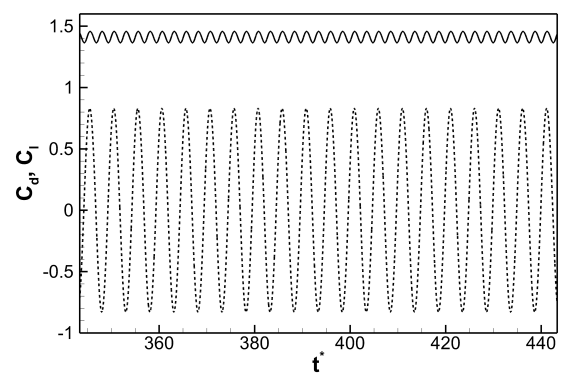

(a) $R e=200$

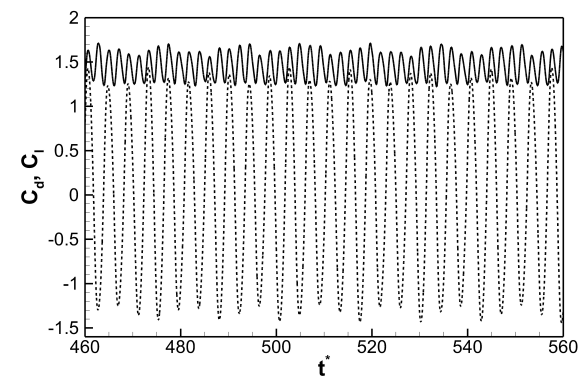

(b) $R e=1000$

Figure 7: Time history of the drag and the lift coefficients $C_{d}$ and $C_{l}, C_{d}:-\cdots ; C_{l}$ : - .

Table 5: Comparison of coefficients $C_{d}$ and $S t$ for $R e=1000$.

\begin{tabular}{lllll}
\hline & The relaxed MDF-IBM & Haeri and Shrimpton [4] & Mittal et al. [39] & Apte et al. [40] \\
\hline$C_{d}$ & 1.43 & 1.48 & 1.45 & 1.50 \\
$S t$ & 0.235 & 0.238 & 0.231 & 0.238 \\
\hline
\end{tabular}

\subsection{Flow past multiple obstacles}

This section applies the relaxed MDF-IBM in a flow past several fixed obstacles. The size of the computational domain and the boundary conditions are the same as these in the previous section. Here a circular cylinder and two diamonds of different sizes are embedded. The circular cylinder with a diameter $D=50 \delta x$ is fixed at the location $(9 D, 10 D)$. The diamond is defined with a length $L$ and a height $H$ as illustrated in Fig. 8 The diamond with size $(H, L)=(60,60)$ is fixed at $(10 D, 11 D)$ and the narrow diamond with size $(H, L)=(10,100)$ at $(10 D, 9 D)$. The Reynolds number based on the diameter $D$ and the inlet velocity $U_{0}=0.05$ is 100 .

Fig. 9 shows the variations of $\|\mathbf{A}\|^{-1}$ of different delta functions versus the relative hight $H / L$ and $\delta s \approx \delta x$. It shows that as $H / L$ grows from 0.1 to 1.0 , $\|\mathbf{A}\|^{-1}$ increases from 1.42 to 2.31 for $\Phi_{4 r}$ and $\Phi_{4 c}$, and from 1.52 to 2.48 for $\Phi_{4 s}$. Thus, compared to $\delta s$, the shape of boundary has a more significant influence on the value of $\|\mathbf{A}\|^{-1}$. As discussed in the previous section, the average relaxation parameter of a circular cylinder over $\delta s$ is $\omega=2.6$. If $\omega=2.6$, the iteration of 


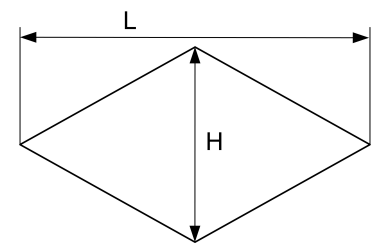

Figure 8: The sketch of diamond immersed in a viscous flow.

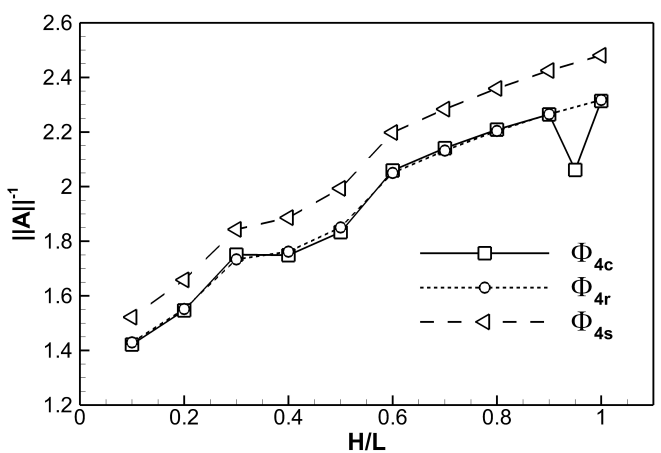

Figure 9: Variation of the value of $\|\mathbf{A}\|^{-1}$ with the shape parameter $H / L$.

Here the delta function $\Phi_{4 r}$ is employed. Because the distances between any two obstacles exceed $4 \delta x$, the estimated optimal relaxation parameters corresponding to the cylinder, the big diamond, and the narrow diamond are $(2.60,2.31,1.42)$, respectively. Fig. 11 displays the variation of $E$ with the non- 


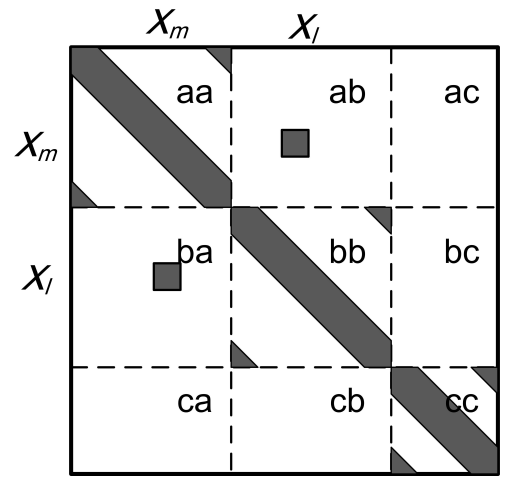

Figure 10: The sparsity pattern of matrix A corresponding to three objects $(a, b, c)$, non-zero element:

dimensional time $t^{*}$, where $\omega=(1.42,1.42,1.42)$ is the overall relaxation parameter of matrix $\mathbf{A}$ and $\omega=(1.0,1.0,1.0)$ represents the traditional MDF-IBM. The case with $\omega=(2.60,2.31,1.42)$ and $N_{i t r}=1$ produces the smallest error $E$, which is equivalent to the traditional MDF-IBM with $N_{i t r}=3$. The vorticity contour of the flow past the three obstacles can be seen in Fig. 12 .

\subsection{Particle in a linear shear flow}

For moving objects, the matrix A should be updated in every time step as the relative locations between the no-slip boundaries and underlying lattices keep changing. Therefore, the movement of a circular particle in a linear shear flow is considered here to explore the influence of moving objects on the value

330 of $\|\mathbf{A}\|^{-1}$. Fig. 13 shows the sketch of the neutrally buoyant particle in a linear shear flow. The parameters for this simulation are set to $\rho_{p}=\rho_{f}=1.0$, $W=4 D$ and $L=100 D$ 41]. The upper and the lower walls of the channel are set as half-bounce-back boundaries moving with the same magnitude but in the opposite directions, and the inlet and the outlet are periodic. The Reynolds number is $R e=U_{w} W / \nu=40$ with $W=80$ and $U_{w}=0.0125$ in lattice units. Initially, the particle is placed at the position $(10 D, D)$ of a resting flow field. Because the centreline is an equilibrium state, once the particle is released, it will move upward and migrate to the centreline gradually with a dimensionless 


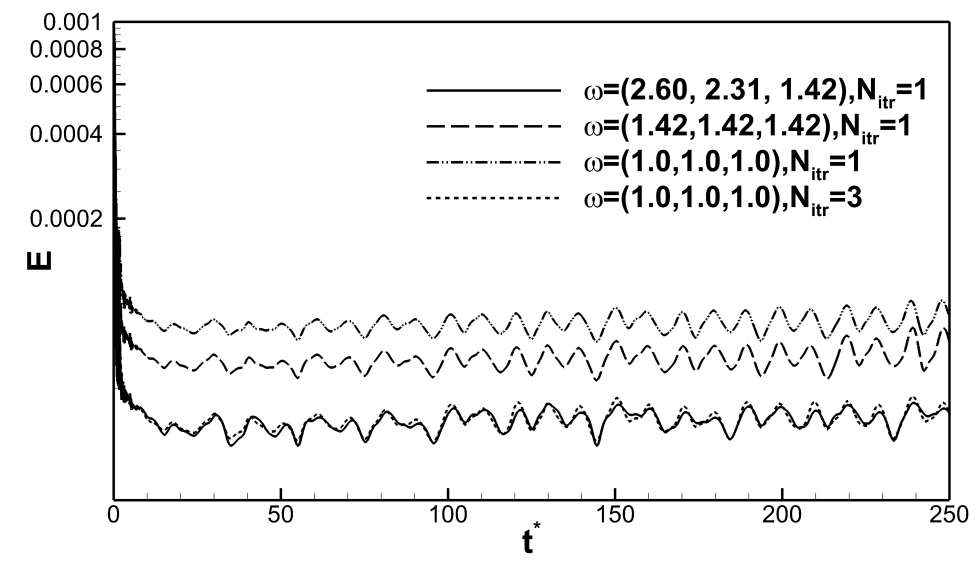

Figure 11: Time history of the numerical error $E$ of flow past three obstacles, $\omega=(1.0,1.0,1.0)$ corresponds to the traditional MDF-IBM.

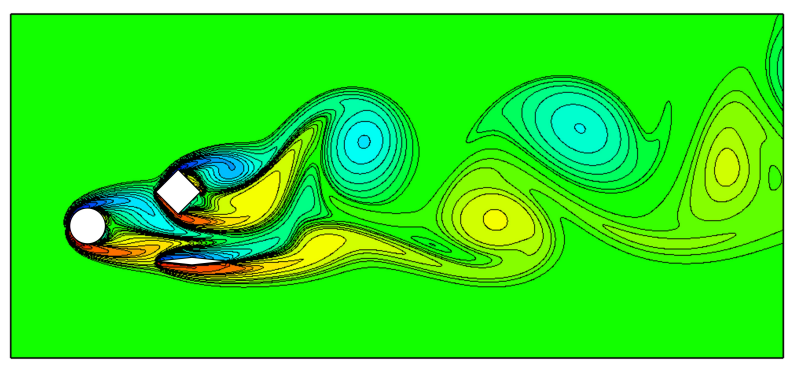

Figure 12: The vorticity contour of flow past three obstacles: a cylinder, a big diamond and a narrow diamond. 


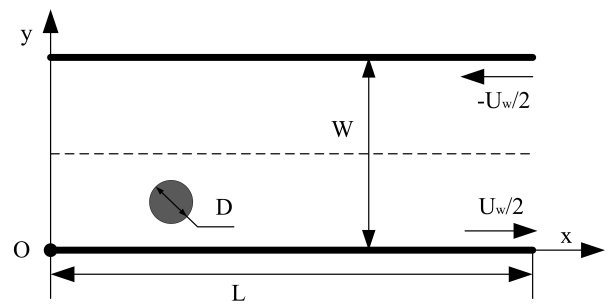

Figure 13: The sketch of a particle in a linear shear flow between walls.

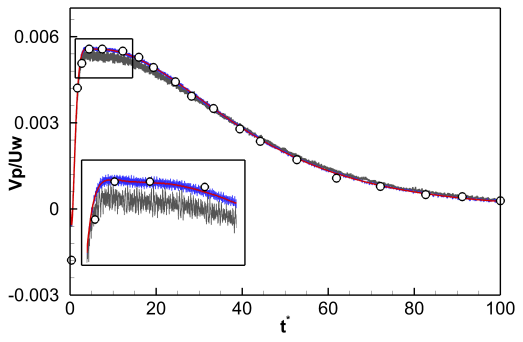

(a)

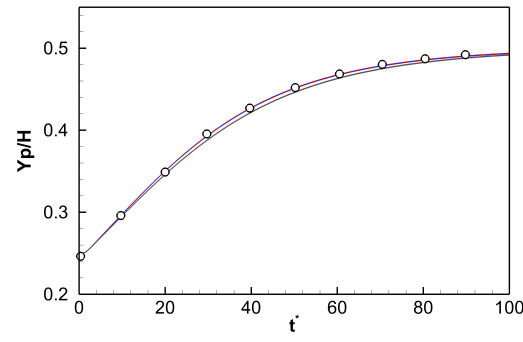

(b)

Figure 14: Variations of the vertical velocity and displacement of the particle in a shear flow, results from Niu et al. [41]: o; present results with delta functions $\Phi_{4 r}:-; \Phi_{4 c}:----; \Phi_{4 s}$ :

time $t^{*}=t U_{w} / W$. et al. 41], where a good agreement is achieved between the results from the delta functions $\Phi_{4 r}$ and $\Phi_{4 c}$. However, the maximum vertical velocity obtained from $\Phi_{4 s}$ is slightly lower. It should be noted that in Fig. 14a. $V_{p} / U_{w}$ of the delta functions $\Phi_{4 c}$ and $\Phi_{4 s}$ shows an oscillation, while $V_{p} / U_{w}$ of $\Phi_{4 r}$ does not. It turns out that for moving boundaries, the delta function $\Phi_{4 r}$ is the most stable among the delta functions investigated here. Fig. 15 shows the development of $E$ during this process, where for $N_{i t r}=5$, the delta function $\Phi_{4 r}$ works better than the others. In addition, when the iteration times $N_{i t r}$ of $\Phi_{4 r}$ is reduced to 2 and 1 (see Fig. 15), the reduction of $E$ is relatively small. Therefore, the 350 error $E$ with $\omega=\|\mathbf{A}\|^{-1}$ and $N_{i t r}=5$ will be used to assess the performance of the relaxation MDF-IBM with a constant $\omega$. 


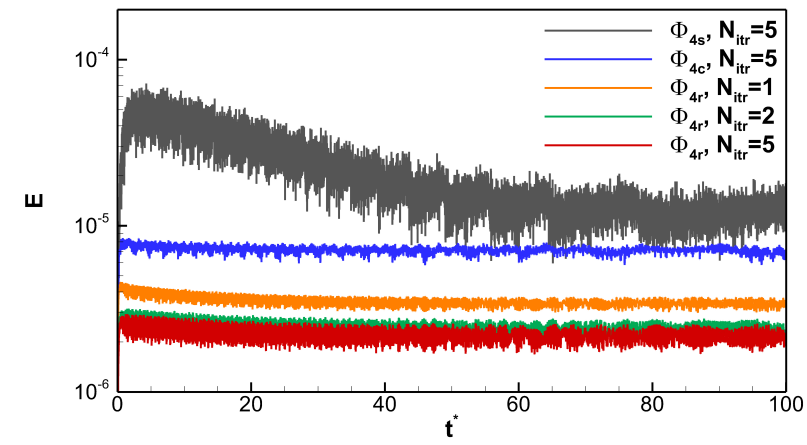

Figure 15: Variations of $E$ as the particle moves in the shear flow, $\omega=\|\mathbf{A}\|^{-1}$.

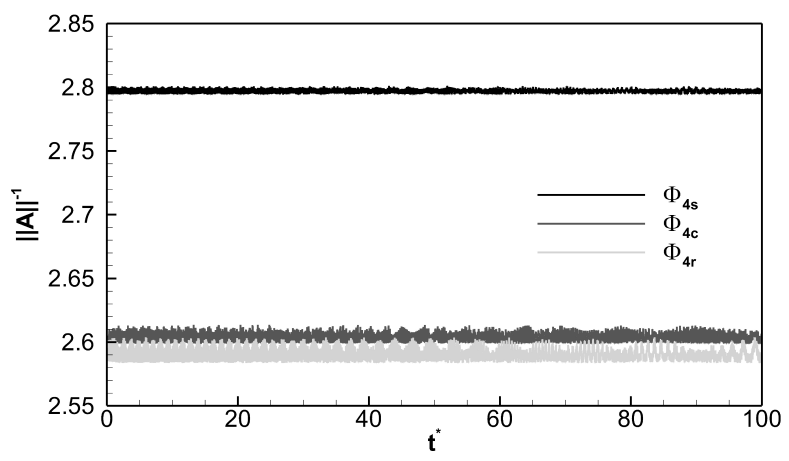

Figure 16: Variations of $\|A\|^{-1}$ as the particle moves in the shear flow.

Fig. 16 shows that when the particle moves across the underlying lattices, $\|\mathbf{A}\|^{-1}$ vibrates in a small interval, approx. $(2.794,2.801)$ for $\Phi_{4 s},(2.599,2.613)$ for $\Phi_{4 c}$, and $(2.583,2.604)$ for $\Phi_{4 s}$. With the constant relaxation parameters $\omega=2.6$ for $\Phi_{4 s}$ and $\Phi_{4 c}, \omega=2.8$ for $\Phi_{4 s}$, the time variations of $E$ are plotted in Fig. 17a. $E$ is slightly higher for the cases of a constant $\omega$ and $N_{i t r}=1$ than those of a variable $\omega$ and $N_{i t r}=5$. It demonstrates that despite the relaxed MDF-IBM that takes $\omega=\|\mathbf{A}\|^{-1}$ and $N_{i t r}=5$ is more accurate, the cases with a constant $\omega$ and $N_{i t r}=1$ can still achieve reasonably accurate no-slip boundary. Fig. 17b shows that the performance of the relaxed MDF-IBM with $N_{i t r}=1$ is slightly better than that of the traditional MDF-IBM with $N_{i t r}=7$. 


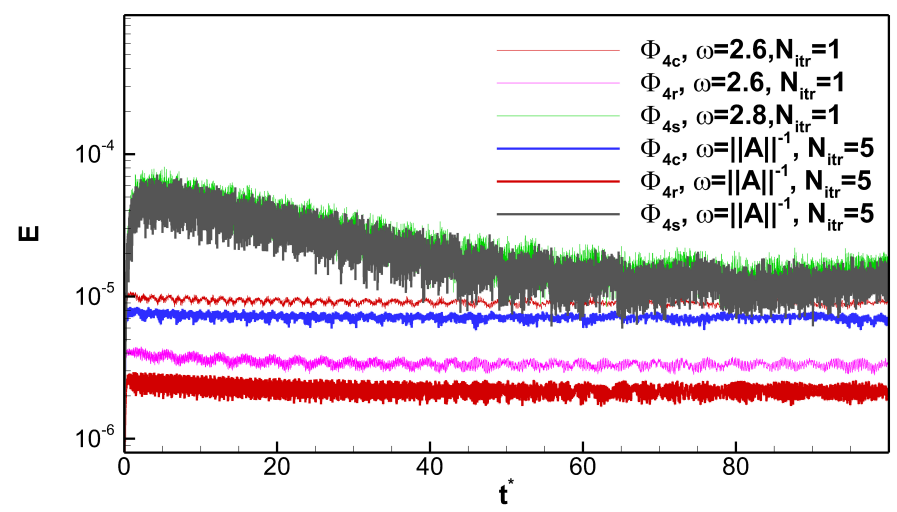

(a)

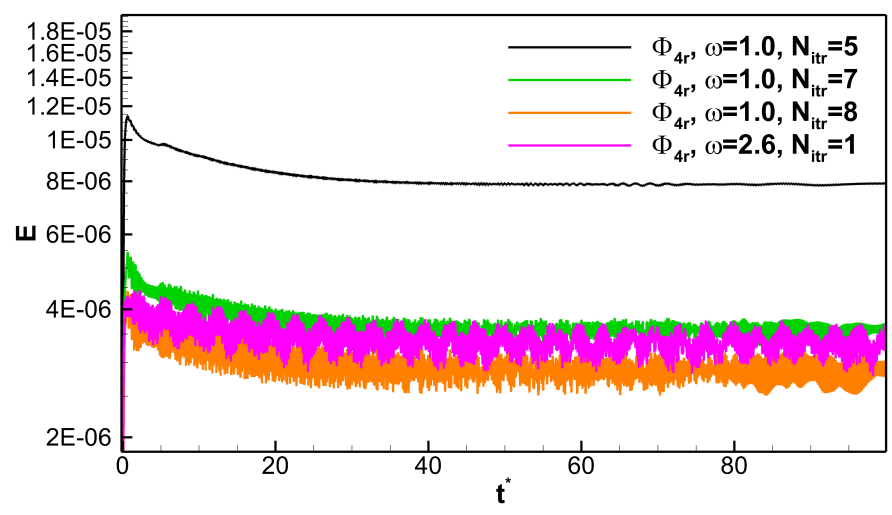

(b)

Figure 17: Variations of $E$ as the particle moves in the shear flow, $\omega=1.0$ corresponds to the traditional MDF-IBM. (For interpretation of the colours in the figures, the reader is referred to the web version of this article.) 


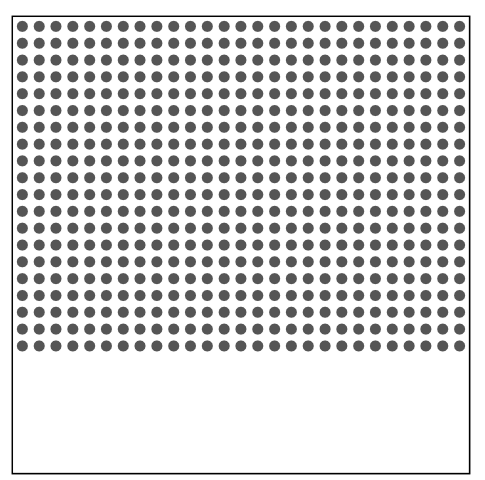

Figure 18: The initial arrangement of 540 particles.

\subsection{The sedimentation of multiple particles in an enclosure}

As discussed in Section 3.2 , the relative location between objects has a significant influence on the structure of matrix A. For the cases with several moving objects, non-zero elements appear in non-diagonal blocks of matrix $\mathbf{A}$ as the objects come close to each other. To study the influence of the relative location between objects on the value of $\|\mathbf{A}\|^{-1}$, multiple circular particles are released in a two-dimensional enclosure filled with a viscous fluid. Initially, the fluid and particles are stationary; then the particles will move under the gravity of $9.81 \mathrm{~m} / \mathrm{s}^{2}$. The size of the cavity is $25.6 \mathrm{~mm} \times 25.6 \mathrm{~mm}$, the diameter of particles is $D=0.625 \mathrm{~mm}$. The fluid density is $\rho_{f}=1.0 \mathrm{~g} / \mathrm{cm}^{3}$, the particle's density $\rho_{p}=1.01 \rho_{f}$. The fluid viscosity is $1 \mathrm{~g} / \mathrm{ms}$. The initial gap between the neighbouring particles in the same row and column is $D / 2$. There are 79 Lagrangian points over the surface of every circular particle. The size of the computational domain is $1024 \times 1024$ in lattice units. Fig. 18 shows the original arrangement of 540 particles.

Our simulations on GPU executes a similar strategy for the single and multiple body movements. The only difference is that the latter requires a reliable collision strategy to model the kinetic energy transfer between the colliding particles. The discrete element method (DEM) 42] [43, which is combined with IB-CLBM in Ref. [23], is used here to resolve the collision of particles with the 
walls and each other. Here, a spring-dashpot model of DEM [44] is employed, where the total contact force $\mathbf{F}_{c}$ exerting on a particle $i$ by the other particles and the walls is

$$
\mathbf{F}_{c}=\sum^{p, \text { wall }}\left(F_{c n} \mathbf{n}+F_{c t} \mathbf{t}\right) .
$$

In this case, considering the aim of preventing the non-physical overlap between particles, the tangential contact force $F_{c t}$, which contributes to the tangential sliding of particles, can be ignored. The normal contact force $F_{c n}$ is calculated from

$$
F_{c n}=k_{n} \delta_{n}+c_{n} v_{r n}, \delta_{n}>0
$$

where $k_{n}$ and $c_{n}$ are the normal spring and damping coefficients, respectively. Collision between the particles $i$ and $j$ occurs when the overlap length $\delta_{n}=$ $380 \quad\left(R_{i}+R_{j}+\epsilon_{c}\right)-\left|\mathbf{X}_{i}-\mathbf{X}_{j}\right|$ is positive, where $\epsilon_{c}$ is a critical distance and $R$ is the radius of particles. The total relative velocity at the contact point of particles is $\mathbf{V}_{r}=\mathbf{U}_{i}-\mathbf{U}_{j}+R_{i} \mathbf{n} \times \boldsymbol{\Omega}_{c i}+R_{j} \mathbf{n} \times \boldsymbol{\Omega}_{c j}$. The relative velocity in normal direction is $v_{r n}=-\mathbf{V}_{r} \cdot \mathbf{n}$. The unit normal vector is $\mathbf{n}=\left(\mathbf{X}_{i}-\mathbf{X}_{j}\right) /\left|\mathbf{X}_{i}-\mathbf{X}_{j}\right|$. The model handles the particle-wall collisions in a similar fashion, where $\delta_{n}=$ ${ }_{385}\left(R_{i}+\epsilon_{c}\right)-\left|\mathbf{X}_{i}-\mathbf{X}_{\text {wall }}\right|, \mathbf{U}_{\text {wall }}=0$, and $\omega_{\text {wall }}=0$. In this work, the values of $k_{n}$ and $c_{n}$ are chosen to yield a theoretical restitution coefficient of $r_{c}=0.98$.

This collision strategy allows overlap of the particles to represent the physical deformation of particles. However, an overlap translates to possible intersections between boundaries, which makes the structure of matrix A experience a dramatic change and hence, a considerable variation of $\left.\|\mathbf{A}\|\right|^{-1}$.

For multi-particle simulations, all the simulations are carried out on a host CPU (Intel(R) Xeon(R) CPU E5-2670 0 @ 2.60GHz) and a device GPU (NVIDIA Tesla K20m, compute capability 3.5, memory size 5GB) with the same computational domain. The code is compiled by NVIDIA C-Compiler (NVCC) with the compilation option (nvcc -arch sm_30 -m 64 -Xptxas -O3 X.cu -o X) and runs on Linux operation system (Red Hat Enterprise Linux Server release 6.3 (Santiago)). It can be seen from Fig. 19 that the time consumed by the functions A1 and A2 grows rapidly as the number of Lagrangian points $N$ increases, 


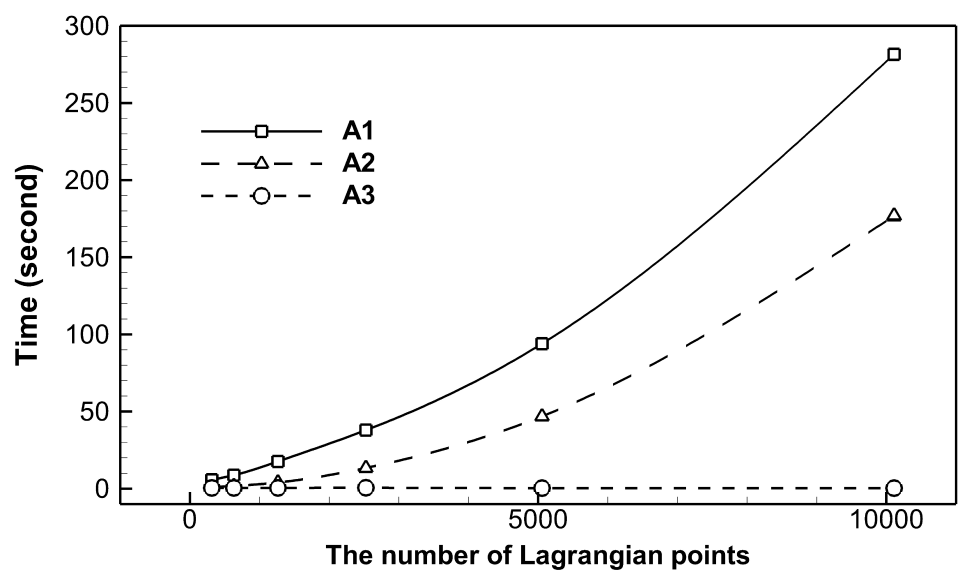

Figure 19: The time consumed by different functions to calculate $\|\mathbf{A}\|^{-1}$ for $10^{4}$ time steps with 256 threads in each block.

while the computational time required to evaluate the function A3 consistently remains negligible. Moreover, the large size of matrix A $N^{2}$ will put significant pressure on the global memory available on the GPU. To be specific, the simulation of 540 particles, which involves 42660 Lagrangian points, results in a matrix $\mathbf{A}$ of size $13.56 \mathrm{~GB}$ in a double-precision floating-point computation. Thus, two/three-particle collision events are considered in Fig. 20 to explore the possibility of determining an appropriate constant relaxation parameter before the simulation. The variation of $\|\mathbf{A}\|^{-1}$ for different delta functions as a function of distance between the particles is presented.

For the two particles that come toward each other, initially, $\|\mathbf{A}\|^{-1}$ values are close to 2.6 and 2.8 respectively, representing the convergent ranges of $(0,5.2)$ and $(0,5.6)$, consistent with the single cylinder in the previous section; then, as the particles come closer to each other, the values drop quickly to 1.3 and 1.5 , representing the convergent ranges of $(0,2.6)$ and $(0,3.0)$. For the three particles arranged in an equilateral triangular pattern, $\|\mathbf{A}\|^{-1}$ experiences one more collapse when a severe overlap happens, where $\|\mathbf{A}\|^{-1}=0.9$ when the distance is $-3.4 \delta x$ for $\Phi_{4 r}$, representing the convergent range of $(0,1.8)$. These two shifts of the convergent range from $(0,5.2)$ to $(0,1.8)$ could cause the sim- 


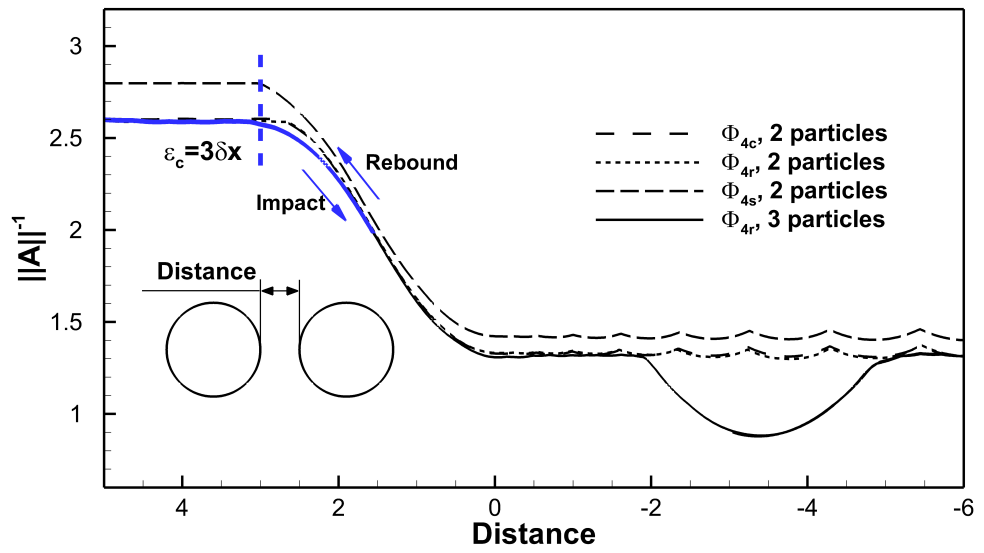

Figure 20: Variations of $\|\mathbf{A}\|^{-1}$ as the particles move toward each other. $\|\mathbf{A}\|^{-1}$ over the collision procession is marked with lines: —and -....

ulation to diverge when any two or three particles come into contact with each other if a constant $\omega=2.6$ or 2.8 is used. Since the smallest $\|\mathbf{A}\|^{-1}$ appears when the severest overlap occurs, the difficulty can be alleviated by increasing the value of $\epsilon_{c}$. For example, in Fig. 20 when the particles come into a distance shorter than $\epsilon_{c}=3 \delta x$, the contact force $\mathbf{F}_{c}$ is exerted to separate the particles.

Two sets of parameters $\epsilon_{c}=3 \delta x$ and $\epsilon_{c}=0$, based on the delta function $\Phi_{4 r}$, are tested in Fig. 21 for the numerical simulation involving multiple moving particles. Fig. 21a shows that with $N_{i t r}=1$ as $\omega$ increases from 2.0 to 2.4, the error $E$ becomes smaller. Moreover, $E$ with $N_{i t r}=1$ and $\omega=2.4$ is similar to that of the traditional MDF-IBM with $N_{i t r}=5$. On the other hand, when $\epsilon_{c}=0$ (see Fig. 21b), $E$ with $N_{i t r}=2$ and $\omega=1.4$ is similar to that of the traditional MDF-IBM with $N_{i t r}=3$. Fig. 22 depicts several snapshots of the sedimentation of 540 particles. In the beginning, the cases with different $\epsilon_{c}$ values show similar movement patterns. Then, the particles fall in a random distribution until settling in the bottom of the container, where it is clear that the particles with a smaller $\epsilon_{c}=0$ form a denser packing.

In this work, the efficiency improvement of the relaxed MDF-IBM part mainly comes from the reduced iteration time when reaching a similar numerical 


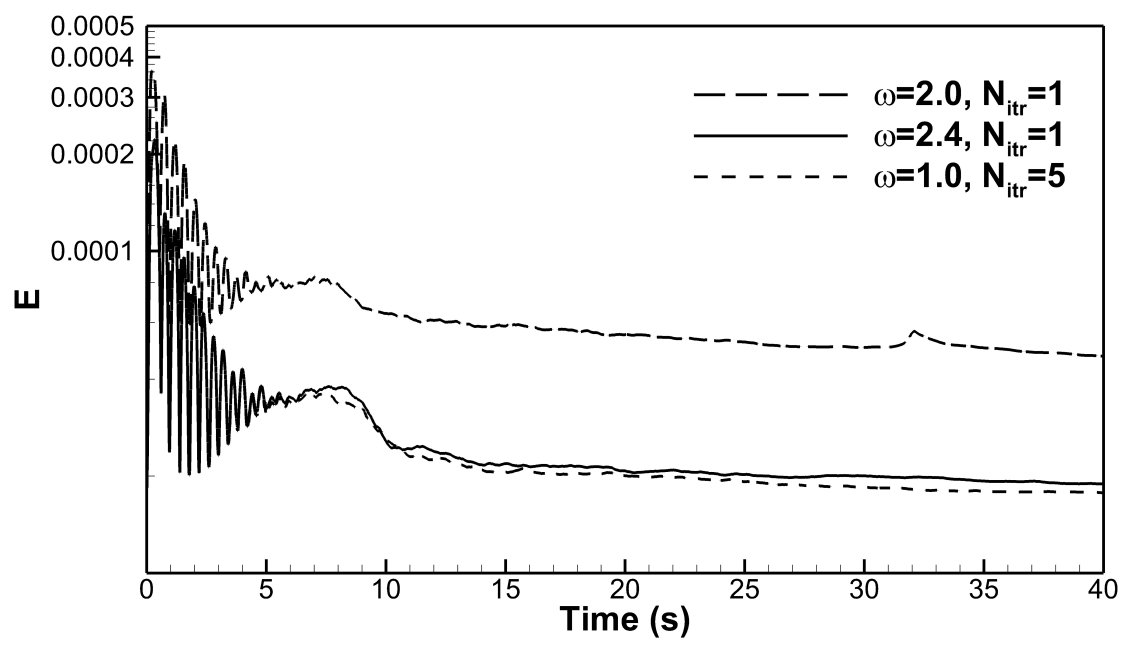

(a) $\epsilon_{c}=3 \delta x$

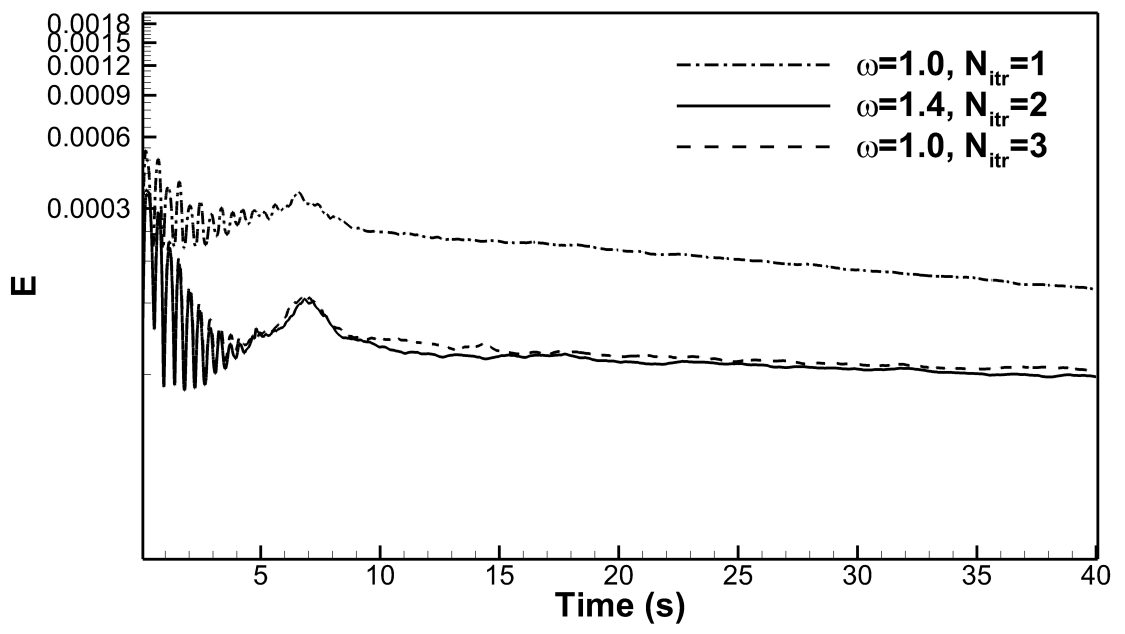

(b) $\epsilon_{c}=0$

Figure 21: Variations of $E$ as the 540 particles fall, $\omega=1.0$ corresponds to the traditional MDF-IBM. 

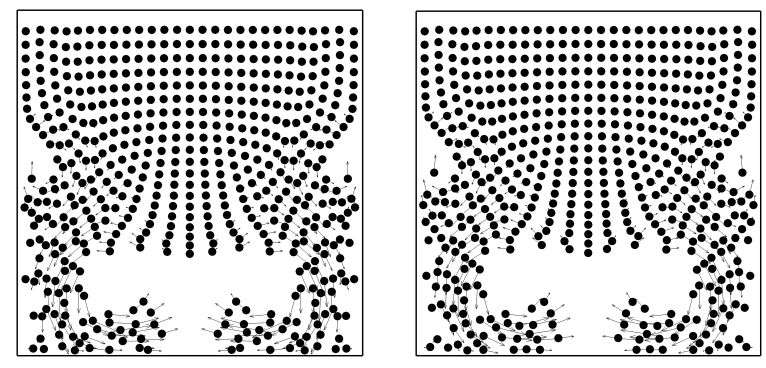

(a) $t=4 \mathrm{~s}, \epsilon_{c}=0$

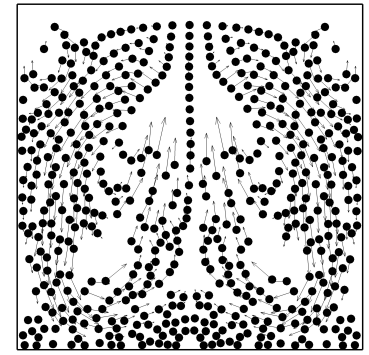

(b) $t=4 \mathrm{~s}, \epsilon_{c}=3 \delta x$

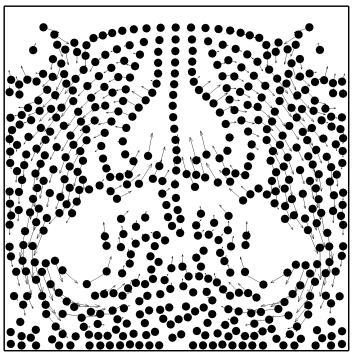

(c) $t=6 \mathrm{~s}, \epsilon_{c}=0$

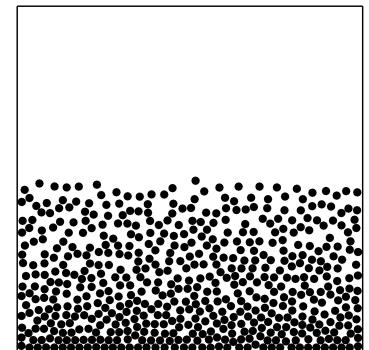

(d) $t=6 \mathrm{~s}, \epsilon_{c}=3 \delta x$

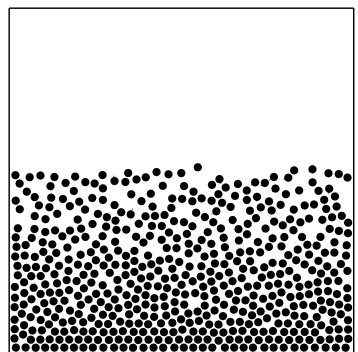

(e) $t=20 \mathrm{~s}, \epsilon_{c}=0$

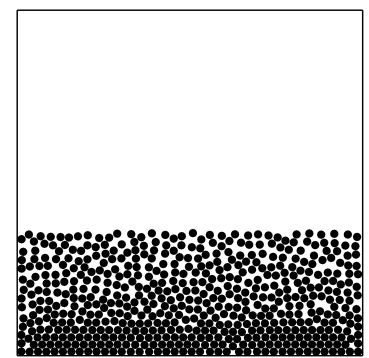

(f) $t=20 \mathrm{~s}, \epsilon_{c}=3 \delta x$

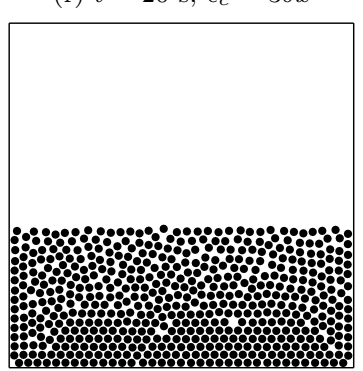

(g) $t=40 \mathrm{~s}, \epsilon_{c}=0$

(h) $t=40 \mathrm{~s}, \epsilon_{c}=3 \delta x$

Figure 22: The sedimentation of 540 particles in an enclosure filled with a viscous fluid. 
in Table 6. The maximum MLPUPS is 9.94 when the relaxed MDF-IBM is used to update 42660 Lagrangian points on GPU. The corresponding MLUPS - million lattices updated per second - is 184.78 based on the computational time 56.75 seconds for the LB solver on a $1024 \times 1024$ lattice.

\section{Conclusion}

In this work, a relaxed MDF-IBM has been proposed with the objective of enforcing a much higher quality no-slip boundary and improving the computational efficiency of MDF-IBM when dealing with fluid-solid interactions on a 


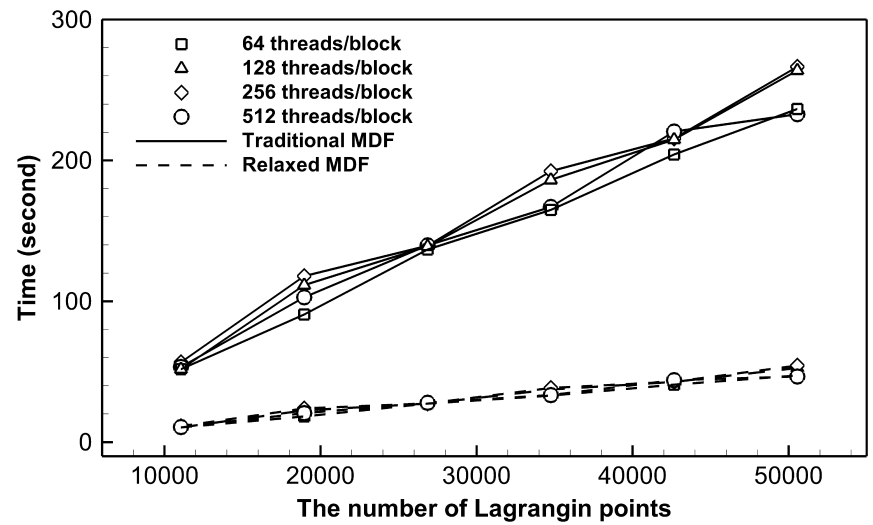

(a) The time consumed for $1 \times 10^{4}$ time steps.

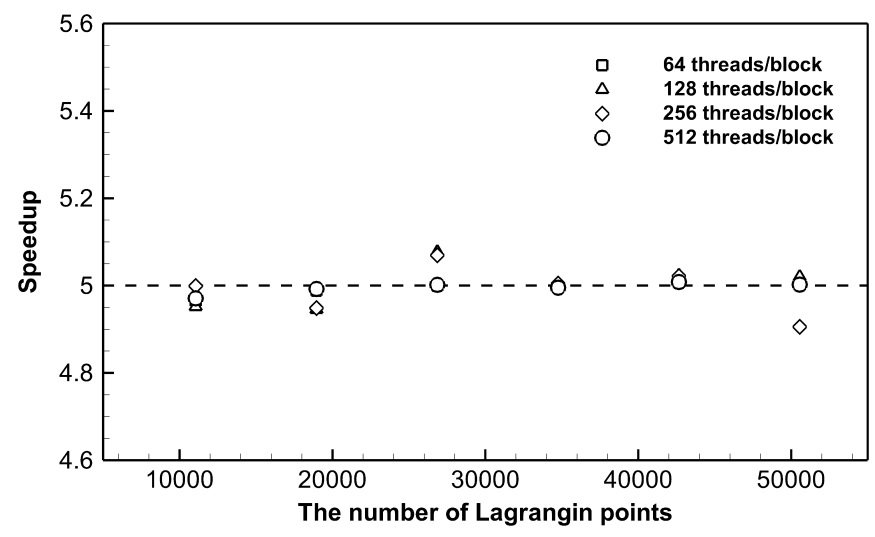

(b) The speed-ups.

Figure 23: The computational performance of the traditional MDF-IBM with $N_{i t r}=5$ and the relaxed MDF-IBM part with $N_{i t r}=1$ on GPU. 
Table 6: The speed-up of the relaxed and the traditional MDF-IBM methods (GPU vs. CPU.)

\begin{tabular}{|c|c|c|c|c|c|c|c|}
\hline \multicolumn{2}{|c|}{ Number of Lagrangian points } & 11060 & 18960 & 26860 & 34760 & 42660 & 50560 \\
\hline \multicolumn{8}{|c|}{ The relaxed MDF-IBM with $N_{i t r}=1$} \\
\hline \multirow{2}{*}{$\mathrm{CPU}$} & Time (second) & 185.9 & 329.7 & 475.6 & 575.1 & 714.8 & 771.7 \\
\hline & MLPUPS & 0.59 & 0.58 & 0.56 & 0.60 & 0.60 & 0.66 \\
\hline \multirow{2}{*}{ GPU } & Time (second) & 11.3 & 23.8 & 27.5 & 38.4 & 42.9 & 54.3 \\
\hline & MLPUPS & 9.76 & 7.96 & 9.77 & 9.04 & 9.94 & 9.31 \\
\hline \multicolumn{2}{|c|}{ The speed-up } & 16.4 & 13.8 & 17.3 & 15.0 & 16.7 & 14.2 \\
\hline \multicolumn{8}{|c|}{ The traditional MDF-IBM with $N_{i t r}=5$} \\
\hline \multirow{2}{*}{$\mathrm{CPU}$} & Time (second) & 831.6 & 1422.0 & 2110.9 & 2624.9 & 3249.7 & 3813.4 \\
\hline & MLPUPS & 0.13 & 0.13 & 0.13 & 0.13 & 0.13 & 0.13 \\
\hline \multirow{2}{*}{ GPU } & Time (second) & 56.6 & 117.9 & 139.4 & 192.4 & 215.5 & 266.5 \\
\hline & MLPUPS & 1.95 & 1.61 & 1.93 & 1.81 & 1.98 & 1.90 \\
\hline \multicolumn{2}{|c|}{ The speed-up } & 14.7 & 12.1 & 15.1 & 13.6 & 15.1 & 14.3 \\
\hline
\end{tabular}

single GPU. More specifically, the convergence rate of the MDF-IBM is accelerated significantly, where the iteration number reduces at least between 3 and 7 times for different problems considered in this work.

The traditional method is analysed and found to be equivalent to a relaxed linear system iteration with a constant relaxation parameter of 1.0. When employing an estimated optimal relaxation parameter in a typical case of flow around a fixed cylinder, no-slip boundary is fulfilled after only one iteration and reaches a much smaller deviation. As it is computationally expensive to update the relaxation parameter for moving boundaries in every time step, the possibility of using an appropriate constant relaxation parameter is explored. The contributions of different factors, including the delta functions, the spacing size between the Lagrangian points $\delta s$, the shape and relative location of no-slip boundaries, the displacement of moving boundary over underlying lattices, are studied in the four typical cases, i.e. the flow around a fixed cylinder, flow cross multiple obstacles, the particle in a linear shear flow, and the sedimentation 
of many particles in an enclosure. It turns out that the first three factors are the most influential. Thus, it is feasible to predetermine an appropriate constant relaxation parameter according to the used delta function and the no-slip boundary shape, and also by predicting the possible relative movement between boundaries.

In this work, the efficiency improvement of the algorithm firstly comes from the reduction of iteration time when reaching a similar deviation. Secondly, the employment of a predetermined constant relaxation parameter simplifies the algorithm which minimises the computational burden. Moreover, the parallel implementation of the relaxed MDF-IBM on a single GPU is described in detail. The pseudo-codes of calculating the estimated optimal parameter are presented in this work. The potential of our relaxed MDF-IBM for solving complex fluid-solid interactions efficiently with GPU parallel implementation has been demonstrated. Compared to the traditional MDF-IBM, the relaxed method has the following advantages:

1) improved efficiency and accuracy for no-slip boundaries in a viscous fluid.

495 2) a simple and appropriate constant relaxation parameter for moving boundaries.

3) can be adopted for use in conjunction with the traditional MDF-IBM.

\section{Acknowledgment}

This work is supported by the National Natural Science Foundation of China

(Grant No. 51709229, 51879220 and 61803306); National Key Research and Development Program of China (Grant No. 2016YFC0301300); Natural Science Basic Research Plan in Shaanxi Province of China (Grant No. 2018JQ5092).

\section{References}

\section{References}

[1] Peskin, C.S.. Numerical analysis of blood flow in the heart. Journal of Computational Physics 1977;25(3):220-252. 
[2] Haeri, S., Shrimpton, J.S.. On the application of immersed boundary, fictitious domain and body-conformal mesh methods to many particle multiphase flows. International Journal of Multiphase Flow 2012;40(March 2017):38-55.

[3] Haeri, S., Shrimpton, J.S.. A mesoscopic description of polydispersed particle laden turbulent flows. Progress in Energy and Combustion Science 2011;37(6):716-740.

[4] Haeri, S., Shrimpton, J.S.. A new implicit fictitious domain method for the simulation of flow in complex geometries with heat transfer. Journal of Computational Physics 2013;237:21-45.

[5] Haeri, S., Shrimpton, J.S.. Fully resolved simulation of particle deposition and heat transfer in a differentially heated cavity. International Journal of Heat and Fluid Flow 2014;50(December):1-15.

[6] Wang, Z., Fan, J., Luo, K.. Combined multi-direct forcing and immersed boundary method for simulating flows with moving particles. International Journal of Multiphase Flow 2008;34(3):283-302.

[7] Noble, D.R., Torczynski, J.R.. A Lattice-Boltzmann Method for Partially Saturated Computational Cells. International Journal of Modern Physics C 1998;09(08):1189-1201.

[8] Krause, M.J., Klemens, F., Henn, T., Trunk, R., Nirschl, H.. Particle flow simulations with homogenised lattice Boltzmann methods. Particuology 2017;34:1-13.

[9] Trunk, R., Marquardt, J., Thäter, G., Nirschl, H., Krause, M.J.. Towards the simulation of arbitrarily shaped 3D particles using a homogenised lattice Boltzmann method. Computers and Fluids 2018;172:621-631.

[10] Ladd, A.J.C.. Numerical simulations of particulate suspensions via a discretized Boltzmann equation. Part 1. Theoretical foundation. Journal of Fluid Mechanics 1994;271:285. 
[11] Aidun, C.K., Clausen, J.R.. Lattice-Boltzmann Method for Complex Flows. Annual Review of Fluid Mechanics 2010;42(1):439-472.

[12] Inamuro, T.. Lattice Boltzmann methods for moving boundary flows. Fluid Dynamics Research 2012;44(2):024001.

[13] Chen, S., Doolen, G.D.. Lattice Boltzmann Method. Annual Review of

[14] Geier, M., Greiner, A., Korvink, J.G.. Cascaded digital lattice Boltzmann automata for high Reynolds number flow. Physical Review E - Statistical, Nonlinear, and Soft Matter Physics 2006;73(6):1-10.

[15] Ning, Y., Premnath, K.N., Patil, D.V.. Numerical study of the properties

[19] Minami, K., Suzuki, K., Inamuro, T.. Free flight simulations of a dragonfly-like flapping wing-body model using the immersed boundarylattice Boltzmann method. Fluid Dynamics Research 2015;47(1):15505.

[20] De Rosis, A., Lévêque, E.. Central-moment lattice Boltzmann schemes 560 with fixed and moving immersed boundaries. Computers and Mathematics with Applications 2016;72(6):1616-1628. 
[21] Kuznik, F., Obrecht, C., Rusaouen, G., Roux, J.J.. LBM based flow simulation using GPU computing processor. Computers and Mathematics with Applications 2010;59(7):2380-2392.

[22] Zhou, H., Mo, G., Wu, F., Zhao, J., Rui, M., Cen, K.. GPU implementation of lattice Boltzmann method for flows with curved boundaries. Computer Methods in Applied Mechanics and Engineering 2012;225-228:65-73.

[23] Zhang, Y., Zhang, Y., Pan, G., Haeri, S.. Numerical study of the particle sedimentation in a viscous fluid using a coupled DEM-IB-CLBM approach. Journal of Computational Physics 2018;368(4):1-20.

[24] Wu, J., Cheng, Y., Zhou, W., Zhang, C., Diao, W.. GPU acceleration of FSI simulations by the immersed boundary-lattice Boltzmann coupling scheme. Computers \& Mathematics with Applications 2016;.

[25] Yang, X., Zhang, X., Li, Z., He, G.W.. A smoothing technique for discrete delta functions with application to immersed boundary method in moving boundary simulations. Journal of Computational Physics 2009;228(20):7821-7836.

[26] Mittal, R., Iaccarino, G.. Immersed Boundary Methods. Annual Review of Fluid Mechanics 2005;37(1):239-261.

[27] Falagkaris, E., Ingram, D., Viola, I., Markakis, K.. PROTEUS: A coupled iterative force-correction immersed-boundary multi-domain cascaded lattice Boltzmann solver. Computers \& Mathematics with Applications 2017;74(10):2348-2368.

[28] Hu, Y., Yuan, H., Shu, S., Niu, X., Li, M.. An improved momentum exchanged-based immersed boundary-lattice Boltzmann method by using an iterative technique. Computers \& Mathematics with Applications 2014;68(3):140-155.

[29] Qian, Y.H., D'Humières, D., Lallemand, P.. Lattice BGK Models for Navier-Stokes Equation. EPL (Europhysics Letters) 1992;17:479-484. 
[37] Benson, M.G., Bellamy-Knights, P.G., Gerrard, J.H., Gladwell, I.. A viscous splitting algorithm applied to low Reynolds number flows round a circular cylinder. Journal of Fluids and Structures 1989;3(5):439-479.

[38] Le, D.V., Khoo, B.C., Lim, K.M.. An implicit-forcing immersed boundary

[30] Guo, Z., Zheng, C., Shi, B.. Discrete lattice effects on the forcing term in the lattice Boltzmann method. Physical Review E - Statistical, Nonlinear, and Soft Matter Physics 2002;65(4):1-6.

[31] Suzuki, K., Inamuro, T.. Effect of internal mass in the simulation of a moving body by the immersed boundary method. Computers and Fluids 2011;49(1):173-187.

[32] Wu, J., Shu, C.. Implicit velocity correction-based immersed boundarylattice Boltzmann method and its applications. Journal of Computational Physics 2009;228(6):1963-1979.

[33] Suzuki, K., Inamuro, T.. A higher-order immersed boundary-lattice Boltzmann method using a smooth velocity field near boundaries. Computers and Fluids 2013;76:105-115.

[34] He, X., Doolen, G.. Lattice Boltzmann Method on Curvilinear Coordinates System: Flow around a Circular Cylinder. Journal of Computational Physics 1997;134(2):306-315.

[35] Calhoun, D.. A Cartesian grid method for solving the two-dimensional streamfunction-vorticity equations in irregular regions. Journal of Computational Physics 2002;176(2):231-275.

[36] Braza, M., Chassaing, P., Minh, H.H.. Numerical study and physical analysis of the pressure and velocity fields in the near wake of a circular cylinder. Journal of Fluid Mechanics 1986;165(-1):79.

method for simulating viscous flows in irregular domains. Computer Methods in Applied Mechanics and Engineering 2008;197(25-28):2119-2130. 
[39] Mittal, R., Dong, H., Bozkurttas, M., Najjar, F., Vargas, A., von Loebbecke, A.. A versatile sharp interface immersed boundary method for incompressible flows with complex boundaries. Journal of Computational Physics 2008;227(10):4825-4852.

[40] Apte, S.V., Martin, M., Patankar, N.A.. A numerical method for fully resolved simulation (FRS) of rigid particle-flow interactions in complex flows. Journal of Computational Physics 2009;228(8):2712-2738.

[41] Niu, X.D., Shu, C., Chew, Y.T., Peng, Y.. A momentum exchange-based immersed boundary-lattice Boltzmann method for simulating incompressible viscous flows. Physics Letters, Section A: General, Atomic and Solid State Physics 2006;354(3):173-182.

[42] Haeri, S., Wang, Y., Ghita, O., Sun, J.. Discrete element simulation and experimental study of powder spreading process in additive manufacturing. Powder Technology 2017;306(November):45-54.

[43] Haeri, S.. Optimisation of blade type spreaders for powder bed preparation in Additive Manufacturing using DEM simulations. Powder Technology 2017;321(August):94-104.

[44] Luding, S.. Introduction to discrete element methods: Basic of contact force models and how to perform the micro-macro transition to continuum theory. European Journal of Environmental and Civil Engineering 2008;12(7-8):785-826. 